\title{
THE MEGAMASER COSMOLOGY PROJECT. III. ACCURATE MASSES OF SEVEN SUPERMASSIVE BLACK HOLES IN ACTIVE GALAXIES WITH CIRCUMNUCLEAR MEGAMASER DISKS
}

\author{
C. Y. Kuo ${ }^{1}$, J. A. Braatz ${ }^{2}$, J. J. Condon ${ }^{2}$, C. M. V. Impellizzeri² ${ }^{2}$ K. Y. Lo ${ }^{2}$, I. ZaW $^{3,4}$, M. Schenker $^{5}$, C. Henkel ${ }^{6}$, \\ M. J. REID ${ }^{7}$, AND J. E. GREENE ${ }^{8}$ \\ ${ }^{1}$ Department of Astronomy, University of Virginia, Charlottesville, VA 22904, USA \\ ${ }^{2}$ National Radio Astronomy Observatory, 520 Edgemont Road, Charlottesville, VA 22903, USA \\ ${ }^{3}$ New York University Abu Dhabi, Abu Dhabi, UAE \\ ${ }^{4}$ The Center for Cosmology and Particle Physics, New York University, New York, NY 10003, USA \\ ${ }^{5}$ Department of Astronomy, California Institute of Technology, CA 91125, USA \\ ${ }^{6}$ Max-Planck-Institut für Radioastronomie, Auf dem Hügel 69, 53121 Bonn, Germany \\ ${ }^{7}$ Harvard-Smithsonian Center for Astrophysics, 60 Garden Street, Cambridge, MA 02138, USA \\ ${ }^{8}$ Department of Astrophysical Sciences, Princeton University, Princeton, NJ 08544, USA \\ Received 2010 August 12; accepted 2010 November 9; published 2010 December 28
}

\begin{abstract}
Observations of $\mathrm{H}_{2} \mathrm{O}$ masers from circumnuclear disks in active galaxies for the Megamaser Cosmology Project (MCP) allow accurate measurement of the mass of supermassive black holes $(\mathrm{BH})$ in these galaxies. We present the Very Long Baseline Interferometry images and kinematics of water maser emission in six active galaxies: NGC 1194, NGC 2273, NGC 2960 (Mrk 1419), NGC 4388, NGC 6264 and NGC 6323. We use the Keplerian rotation curves of these six megamaser galaxies, plus a seventh previously published, to determine accurate enclosed masses within the central $\sim 0.3 \mathrm{pc}$ of these galaxies, smaller than the radius of the sphere of influence of the central mass in all cases. We also set lower limits to the central mass densities of between $0.12 \times 10^{10}$ and $61 \times 10^{10} M_{\odot}$ $\mathrm{pc}^{-3}$. For six of the seven disks, the high central densities rule out clusters of stars or stellar remnants as the central objects, and this result further supports our assumption that the enclosed mass can be attributed predominantly to a supermassive $\mathrm{BH}$. The seven $\mathrm{BHs}$ have masses ranging between $0.75 \times 10^{7}$ and $6.5 \times 10^{7} M_{\odot}$, with the mass errors dominated by the uncertainty of the Hubble constant. We compare the megamaser BH mass determination with BH mass measured from the virial estimation method. The virial estimation $\mathrm{BH}$ mass in four galaxies is consistent with the megamaser BH mass, but the virial mass uncertainty is much greater. Circumnuclear megamaser disks allow the best mass determination of the central $\mathrm{BH}$ mass in external galaxies and significantly improve the observational basis at the low-mass end of the $M-\sigma_{\star}$ relation. The $M-\sigma_{\star}$ relation may not be a single, low-scatter power law as originally proposed. MCP observations continue and we expect to obtain more maser $\mathrm{BH}$ masses in the future.
\end{abstract}

Key words: accretion, accretion disks - galaxies: active - galaxies: ISM - galaxies: nuclei - galaxies: Seyfert

Online-only material: machine-readable table

\section{INTRODUCTION}

The primary goal of the Megamaser Cosmology Project (MCP; Braatz et al. 2009; Reid et al. 2009a; Braatz et al. 2010) is to determine the Hubble constant $H_{0}$ to $\sim 3 \%$ accuracy in order to constrain the equation-of-state parameter $w$ of dark energy. The key to achieving this goal is to measure accurate distances to galaxies well into the Hubble flow $(50-200 \mathrm{Mpc})$. A proven method to measure accurate angular-diameter distances involves sub-milliarcsecond resolution imaging of $\mathrm{H}_{2} \mathrm{O}$ maser emission from sub-parsec circumnuclear disks at the center of active galaxies, a technique established by the study of NGC 4258 with Very Long Baseline Interferometry (VLBI; Herrnstein et al. 1999). Throughout the paper, we will refer to such circumnuclear disks as "megamaser disks" and galaxies that contain such megamaser disks as "megamaser galaxies." As the MCP discovers and images megamaser disks, an important result, in addition to the distance determination, is the accurate measurement of the enclosed masses of the massive dark objects (MDOs; Kormendy \& Richstone 1995; Richstone et al. 1998) at the centers of these megamaser galaxies. We assume that the MDOs are black holes (BHs) and justify this assumption in Section 4.

The VLBI plays a crucial role in measuring BH masses with high precision especially in galaxies with lighter supermassive BHs (i.e., $M_{\bullet} \sim 10^{6}-10^{7} M_{\odot}$ ). The critical advantage provided by VLBI is an angular resolution two orders of magnitude higher than the best optical resolution. For any given galaxy with a nearly constant central mass density of stars, $M_{\mathrm{BH}}$ is proportional to $R_{\mathrm{inf}}^{3}$, where $R_{\mathrm{inf}}$ is the radius of the gravitational sphere of influence of BH (Barth et al. 2004). So, a factor of 100 increase in resolution permits measurements of masses up to $10^{6}$ times smaller. Similarly, the central density limits that can be set are up to $10^{6}$ times higher, high enough to rule out extremely dense star clusters as the MDOs based on dynamical argument (see Section 4) in most megamaser disks presented here.

Megamaser disks, such as the archetypal one in NGC 4258, are usually found in the centers of Seyfert 2 galaxies. Because maser emission is beamed and long path lengths are required for strong maser amplification, the megamaser disks are observable only if the disk is close to edge-on. In NGC 4258 the disk is inclined $\sim 82^{\circ}$ and its rotation curve is Keplerian to better than $1 \%$ accuracy, which makes the $\mathrm{BH}$ mass determination robust with very few assumptions. The megamaser disks are typically smaller $(r \sim 0.2 \mathrm{pc}$ in NGC 4258$)$ than the gravitational sphere of influence of their supermassive BHs $(r \sim 1 \mathrm{pc}$ in NGC 4258); this guarantees that the gravitational potential is dominated by the central mass. Applying dynamical arguments to the extremely high mass density $\rho_{0} \sim 10^{11} M_{\odot} \mathrm{pc}^{-3}$ within the NGC 4258 maser disk can rule out a dense cluster of stars or stellar remnants for most of the central dark mass (Maoz 
1995), implying that the measured central mass is dominated by a BH.

The megamaser disk method for estimating $\mathrm{BH}$ masses has some practical limitations. First, finding megamaser disks is difficult, partly because detectable disks need to be nearly edge-on. Only eight BH masses have been published based on measurements of megamasers: NGC 1068 (Greenhill et al. 1996), NGC 2960 (Mrk 1419; Henkel et al. 2002; not based on VLBI), NGC 3079 (Kondratko et al. 2005), NGC 3393 (Kondratko et al. 2008), UGC 3789 (Reid et al. 2009a; MCP Paper I), NGC 4258 (Herrnstein et al. 1999), NGC 4945 (Greenhill et al. 1997), and Circinus (Greenhill et al. 2003). Second, some rotation curves are significantly flatter than Keplerian, e.g., NGC 1068, NGC 3079, and IC 1481 (Mamyoda et al. 2009). The origin of the flatter rotation curves in these galaxies is unclear. It could be caused by self-gravity of a massive disk or the presence of a nuclear cluster, in which case the enclosed mass would be larger than the BH mass, or it could be caused by radiation pressure, in which case the enclosed mass would be smaller than the BH mass (Lodato \& Bertin 2003). Without fully understanding the causes of the flatter rotation curves and making correct modeling for these megamaser disks, accurate $\mathrm{BH}$ mass measurement would be difficult. Therefore, the key to measuring reliable $\mathrm{BH}$ masses is to find well-defined, edge-on megamaser disks with Keplerian rotation curves.

Over the past two decades, there has been substantial progress in detecting $\mathrm{BHs}$ and constraining their masses, especially in quiescent or mildly active nearby galaxies (Kormendy \& Richstone 1995; Kormendy \& Gebhardt 2001; Kormendy 2004; Ferrarese \& Ford 2005). This rapid progress was mainly facilitated by the high angular resolution provided by the Hubble Space Telescope (HST) and by gradually maturing techniques for modeling stellar dynamics of galaxies. The number of $\mathrm{BH}$ detections has increased to the degree that the field has shifted from confirming the existence of BHs to comparing their properties with those of the host galaxies. One of the most discussed relationships found between BHs and host galaxies is the correlation between $\mathrm{BH}$ mass and the velocity dispersion of stars in the bulge (i.e., the $M_{\mathrm{BH}}-\sigma_{\star}$ relation; Ferrarese \& Merritt 2000; Gebhardt et al. 2000; Gültekin et al. 2009, and references therein). It is often suggested that this relation is a manifestation of a causal connection between the formation and evolution of the BH and the bulge. As pointed out by Gebhardt et al. (2000), it is natural to assume that bulges, BHs, and quasars formed, grew, or "turned on" as parts of the same process, in part because the collapse or merger of bulges might provide a rich fuel supply to a centrally located $\mathrm{BH}$. However, the nature of this connection is still not well understood.

Clearly, accurate $\mathrm{BH}$ mass measurements in megamaser galaxies can help to improve our understanding of the $M_{\mathrm{BH}}-\sigma_{\star}$ correlation, especially at the low-mass end. We present highresolution images and rotation curves of megamaser disks in six new galaxies and their $\mathrm{BH}$ masses. In addition, we re-analyze the BH mass in UGC 3789 using the data from Reid et al. (2009a) and present the new measurement here. In Section 2, we present our sample of galaxies, VLBI observations, and data reduction. Section 3 shows the VLBI images and rotation curves of the megamaser disks, followed by our analysis of $\mathrm{BH}$ masses. In Section 4, we rule out compact clusters of stars or stellar remnants as the central dominant masses for the majority of the megamaser disks. In Section 5, we compare results from other mass measuring techniques to our maser masses. The discussion of our main results is presented in Section 6, and we summarize the results in Section 7. A more in-depth discussion of the $M_{\mathrm{BH}}-\sigma_{\star}$ relation, including the new maser $\mathrm{BH}$ masses, is presented in a companion paper by Greene et al. (2010).

\section{THE SAMPLE, OBSERVATION, AND DATA REDUCTION}

\subsection{The Megamaser Disk Sample}

Water masers have so far been detected in $\sim 136$ galaxies. ${ }^{9}$ Most of the megamasers originate in active galactic nuclei (AGNs; Lo 2005) and about $20 \%$ of them show spectra suggestive of emission from sub-parsec scale, edge-on, circumnuclear disks. In the MCP, we are currently conducting VLBI observations of six megamaser disks to determine the angular diameter distances to their host galaxies. While more data are needed to measure their distances accurately, the quality of the VLBI imaging is already good enough to measure precise central $\mathrm{BH}$ masses. In addition to the six galaxies for distance measurements, we also have VLBI data on the megamaser disk in NGC 4388 to measure the central BH mass, even though the data are not suitable for a distance determination. Table 1 lists coordinates, recession velocities, spectral types, and morphological types for these seven galaxies.

\subsection{Observations}

The megamaser galaxies in our sample were observed between 2005 and 2009 with the Very Long Baseline Array (VLBA) ${ }^{10}$ augmented by the $100 \mathrm{~m}$ Green Bank Telescope (GBT) and in most cases the Effelsberg $100 \mathrm{~m}$ telescope. ${ }^{11}$ Table 2 shows the basic observing information including experiment code, date observed, antennas used, and sensitivity.

We observed the megamasers either in a phase-referencing or self-calibration mode. With phase-referencing we perform rapid switching of the telescope pointing between the target source and a nearby $\left(<1^{\circ}\right)$ phase calibrator (every $\sim 50 \mathrm{~s}$ ) to correct phase variations caused by the atmosphere. In a self-calibration observation, we use the brightest maser line(s) to calibrate the atmospheric phase. In both types of observations, we placed "geodetic" blocks at the beginning and end of the observations to solve for atmosphere and clock delay residuals for each antenna (Reid et al. 2009b). For NGC 6323 and NGC 6264, we also placed a geodetic block in the middle of the observations to avoid the zenith transit problem at the GBT and to obtain better calibration. In each geodetic block, we observed 12 to 15 compact radio quasars that cover a wide range of zenith angles, and we measured the antenna zenith delay residuals to $\sim 1 \mathrm{~cm}$ accuracy. These geodetic data were taken in left circular polarization with eight $16 \mathrm{MHz}$ bands that spanned $\sim 370-490 \mathrm{MHz}$ bandwidth centered at a frequency around $22 \mathrm{GHz}$; the bands were spaced in a "minimum redundancy" manner to sample, as uniformly as possible, all frequency differences between intermediate frequency (IF) bands in order to minimize ambiguity in the delay solution (Mioduszewski \& Kogan 2009). We also observed strong compact radio quasars every $\sim 20$ minutes to $2 \mathrm{hr}$ in order to monitor the single-band delays and electronic phase differences among and across the IF bands. The errors of the single-band delays are $<1 \mathrm{~ns}$.

\footnotetext{
9 https://safe.nrao.edu/wiki/bin/view/Main/MegamaserCosmologyProject 10 The VLBA is a facility of the National Radio Astronomy Observatory, which is operated by the Associated Universities, Inc. under a cooperative agreement with the National Science Foundation (NSF).

11 The Effelsberg $100 \mathrm{~m}$ telescope is a facility of the Max-Planck-Institut für Radioastronomie.
} 
Table 1

The Megamaser Sample

\begin{tabular}{|c|c|c|c|c|c|c|c|c|}
\hline Name & $\begin{array}{c}\text { R.A. } \\
(\mathrm{J} 2000)\end{array}$ & $\begin{array}{c}\text { Decl. } \\
(\mathrm{J} 2000)\end{array}$ & $\begin{array}{c}\delta \mathrm{RA} \\
\text { (mas) }\end{array}$ & $\begin{array}{r}\delta \mathrm{DEC} \\
\text { (mas) } \\
\end{array}$ & $\begin{array}{c}V_{\text {sys }} \\
\left(\mathrm{km} \mathrm{s}^{-1}\right)\end{array}$ & $\begin{array}{c}\delta V_{\text {sys }} \\
\left(\mathrm{km} \mathrm{s}^{-1}\right)\end{array}$ & $\begin{array}{c}\text { Spectral } \\
\text { Type }\end{array}$ & $\begin{array}{c}\text { Hubble } \\
\text { Type }\end{array}$ \\
\hline NGC 1194 & $03: 03: 49.10864^{\mathrm{a}}$ & $-01: 06: 13.4743^{a}$ & 0.2 & 0.4 & 4051 & 15 & Sy 1.9 & $\mathrm{SA} 0+$ \\
\hline NGC 2273 & $06: 50: 08.65620^{b}$ & $60: 50: 44.8979^{b}$ & $10^{\mathrm{h}}$ & $10^{\mathrm{h}}$ & 1832 & 15 & Sy 2 & $\mathrm{SB}(\mathrm{r}) \mathrm{a}$ \\
\hline UGC 3789 & $07: 19: 30.9490^{c}$ & $59: 21: 18.3150^{c}$ & $10^{\mathrm{h}}$ & $10^{\mathrm{h}}$ & 3262 & 15 & Sy 2 & (R)SA(r)ab \\
\hline NGC 2960 & $09: 40: 36.38370^{\mathrm{d}}$ & $03: 34: 37.2915^{\mathrm{d}}$ & $10^{\mathrm{h}}$ & $10^{\mathrm{h}}$ & 4945 & 15 & Liner & $\mathrm{Sa}$ ? \\
\hline NGC 4388 & $12: 25: 46.77914^{\mathrm{e}}$ & $12: 39: 43.7516^{\mathrm{e}}$ & 0.4 & 0.3 & 2527 & 1 & Sy 2 & $\mathrm{SA}(\mathrm{s}) \mathrm{b}$ \\
\hline NGC 6264 & $16: 57: 16.12780^{f}$ & $27: 50: 58.5774^{f}$ & 0.3 & 0.5 & 10213 & 15 & Sy 2 & $\mathrm{~S}$ ? \\
\hline NGC 6323 & $17: 13: 18.03991^{\mathrm{g}}$ & $43: 46: 56.7465^{\mathrm{g}}$ & 0.2 & 0.4 & 7848 & 10 & Sy 2 & $\mathrm{Sab}$ \\
\hline
\end{tabular}

Notes. (1) The systemic (recessional) velocities of the galaxies, $V_{\text {sys }}$, listed here are based on the "optical" velocity convention (i.e., no relativistic corrections are made), measured with respect to the LSR. Except NGC 4388, the systemic velocities $V_{\text {sys }}$ are obtained from fitting a Keplerian rotation curve to the observed data as described in Section 3. The uncertainties $\delta V_{\text {sys }}$ given here include both the fitting error and a conservative estimate of the systematic error. The fitting error is typically only about $5 \mathrm{~km} \mathrm{~s}^{-1}$ and the systematic error is from possible deviation of the position of the BH from $\left(\theta_{x}, \theta_{y}\right)=(0,0)$ (see Section 3), which is assumed in our rotation curve fitting. For NGC 4388, we adopt the observed H I velocity from Lu et al. (2003), which has a small but perhaps unrealistic error. (2) The positions of UGC 3789, NGC 1194, NGC 6323, and NGC 4388 refer to the location of maser emission determined from our VLBI phase-referencing observations. The positions of NGC 2273 and NGC 2960 are determined from $K$-band VLA A-array observations of continuum emission from program AB1230 and maser emission from AB1090, respectively. The maser position for NGC 6264 is derived from a phase-referencing observation in the VLBA archival data (project BK114A). (3) The Seyfert types and morphological classifications are from NED.

a The position of the maser spot at $V_{\mathrm{op}}=4684 \mathrm{~km} \mathrm{~s}^{-1}$, where $V_{\mathrm{op}}$ is the "optical" velocity of the maser spot relative to the LSR.

b The position of the radio continuum emission observed at $21867.7 \mathrm{MHz}$ and $21898.9 \mathrm{MHz}$.

c The position of the maser spot at $V_{\mathrm{op}}=2689 \mathrm{~km} \mathrm{~s}^{-1}$.

d The position of the maser spot at $V_{\text {op }}=4476 \mathrm{kms}^{-1}$.

${ }^{\mathrm{e}}$ The position of the maser spot at $V_{\mathrm{op}}=2892 \mathrm{~km} \mathrm{~s}^{-1}$.

f The average position of the masers with velocities from $V_{\text {op }}=10180 \mathrm{kms}^{-1}$ to $V_{\text {op }}=10214 \mathrm{~km} \mathrm{~s}^{-1}$

$\mathrm{g}$ The position of the maser spot at $V_{\mathrm{op}}=7861 \mathrm{~km} \mathrm{~s}^{-1}$.

${ }^{\mathrm{h}}$ For all positions derived from VLA observations, we use 10 mas as the actual position error, rather than use the fitted error from the VLA data, which are only a few mas for these galaxies. The reason is that the systematic error caused by the imperfect tropospheric model of the VLA correlator can be as large as a few to $10 \mathrm{~s}$ mas. A 10 mas position error usually leads to a $\approx 3 \%-5 \%$ error in the BH mass for the megamasers presented here. Note that although the position for UGC 3789 is derived from a VLBI phase-referencing observation with a phase calibrator 2.1 away, the position of this calibrator is derived from a VLA observation. So, we also use 10 mas as the actual position accuracy for UGC 3789.

\subsection{Data Reduction}

We calibrated all the data using the NRAO Astronomical Image Processing System (AIPS). Since the geodetic data and the maser data used different frequency settings, we reduced them separately. For the geodetic data, we first calibrated the ionospheric delays using total electron content measurements (Walker \& Chatterjee 2000) and the Earth Orientation Parameters (EOPs) in the VLBA correlators with the EOP estimates from the US Naval Observatory. ${ }^{12}$ We performed "fringe fitting" to determine the phases, single-band delays, and fringe rates of IF bands of each antenna for every geodetic source, and the multi-band delay of each antenna was determined from these solutions. Finally, we determined the residual tropospheric delay and clock errors for all antennas using the multi-band delays. We applied these corrections to the maser data as described in the next paragraph. In five data sets for NGC 6323, we also made antenna position corrections before the fringe-fitting process when the positions used in the observation deviated from the latest $\mathrm{USNO}^{13}$ solutions by more than $\sim 2 \mathrm{~cm}$.

For the maser data, after the initial editing of bad data, we corrected for ionospheric delay and the EOPs in the same way as for the geodetic data set. We then corrected the sampler bias in the 2-bit correlator. The amplitude calibration was done with the information in the gain table and the system temperature table. We corrected the interferometer delays and phases caused by the effects of diurnal feed rotation (parallactic angle), and applied

\footnotetext{
12 http://gemini.gsfc.nasa.gov/solve_save/usno_finals.erp

$13 \mathrm{http} / / /$ rorf.usno.navy.mil/solutions/
}

the tropospheric delay and clock corrections obtained from the geodetic data afterward. The next step was to perform fringe fitting on one or two scans of the delay calibrators to calibrate the electronic phase offsets among and across IF bands. The frequency axes of the maser interferometer spectra were then shifted to compensate for the changes in source Doppler shifts over the observing tracks.

The final step in calibration was to solve for the atmospheric phase variation by using either phase-referencing or self-calibration. In the phase-referencing mode, we ran the AIPS task CALIB on the phase calibrator to determine the phase correction as a function of time for each individual IF band. In the self-calibration mode, we either selected a single strong maser line or averaged multiple maser lines in narrow ranges of both velocity and space to perform phase calibration. The typical solution interval was $100 \mathrm{~s}$. After the above calibrations, we discarded the phase solutions and the maser data in the time intervals within which the solutions appeared to be randomly scattered in time or had adjacent reference phases exceeding $\sim 50^{\circ}$. The phase solutions were then interpolated and applied to all the maser data. In all phase-referencing observations for NGC 6323, we performed an extra step of self-calibration after the initial phase-referencing calibration, because the phasereferencing calibration alone did not give adequate phase calibration, and significant sidelobes were still visible after CLEAN deconvolution.

The calibrated data from multiple tracks of a single source were combined for imaging. We Fourier transformed the gridded $(u, v)$ data to make images of the masers in all spectral channels of the IF bands that showed maser lines, and we deconvolved the 
Table 2

Observing Parameters

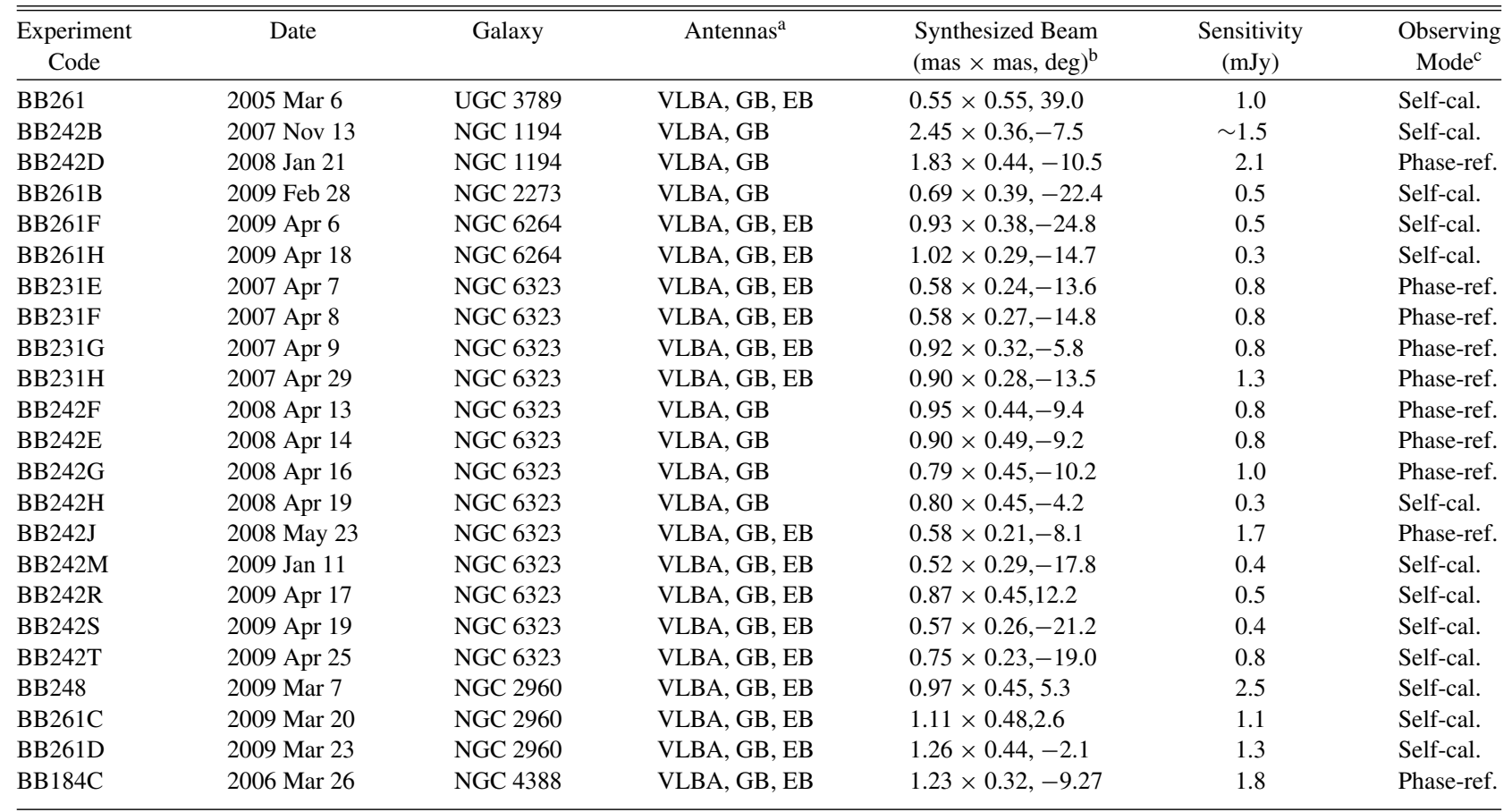

Notes.

a VLBA: Very Long Baseline Array; GB: The Green Bank Telescope of NRAO; EB: Max-Planck-Institut für Radioastronomie 100 m antenna in Effelsberg, Germany.

b Except for program BB184C, this column shows the average FWHM beam size and position angle (P.A.; measured east of north) at the frequency of systemic masers. For BB184C, the FWHM and P.A. are measured at the frequency of red-shifted masers because no systemic maser is detected (FWHM and P.A. differs slightly at different frequencies).

c "Self-cal." means that the observation was conducted in the "self-calibration" mode and "Phase-ref." means that we used the "phase-referencing" mode of observation.

images using CLEAN with a weighting scheme that optimized the position accuracy (e.g., ROBUST $=0$ in the task IMAGR). We fitted the detected maser spots with elliptical Gaussians to obtain the positions and flux densities of individual maser components. In Table 3 we show a representative data set that includes the velocities, positions, and peak intensities of the maser spots in NGC 6264. The data for all galaxies are available in the electronic version of this paper. Note that all galaxies except NGC 4388 and NGC 2273 have at least two tracks of data. Therefore, the peak flux in Table 3 is the flux for the averaged data set. The actual fluxes in an individual epoch can be higher or lower because of variability of the masers.

\subsection{Relativistic Velocity Assignment}

The sub-parsec megamaser disks presented in this paper are in a deep gravitational potential and the majority have recession velocities over $1 \%$ of the speed of light $c$. For this reason, we made both special and general relativistic corrections to the maser velocities in the data before we used the data to analyze the $\mathrm{BH}$ masses. We made the relativistic corrections in the following way.

The observed local standard of rest (LSR) velocities $V_{\text {op }}$ listed in Table 3 are based on the "optical" velocity convention

$$
\frac{V_{\mathrm{op}}}{c}=\left(\frac{v_{0}-v}{v}\right),
$$

where $v$ is the observed frequency and $v_{0}=22.23508 \mathrm{GHz}$, the rest frequency of the $\mathrm{H}_{2} \mathrm{O} 6_{16}-5_{23}$ transition.
Because of gravitational time dilation, the emitting frequency $v_{0}$ of a maser at distance $r$ from a compact object of mass $M$ and the actual observed frequency $v_{\infty}$ for an observer at $r=\infty$ differ by a factor

$$
\frac{v_{0}}{v_{\infty}}=\left(1+\frac{G M}{r c^{2}}\right) .
$$

For a maser in a circular orbit moving at speed $\beta_{\mathrm{m}} c$, balancing the gravitational and centripetal accelerations gives $G M / r^{2}=$ $\left(\beta_{\mathrm{m}} c\right)^{2} / r$, so

$$
\frac{v_{0}}{v_{\infty}}=1+\beta_{\mathrm{m}}^{2}
$$

We multiplied the observed frequency $v$ of each maser line by $\left(1+\beta_{\mathrm{m}}^{2}\right)$ to correct for gravitational time dilation.

The megamaser lines at the systemic velocities were also corrected for a transverse Doppler shift

$$
\frac{v_{0}}{v}=1+\beta_{\mathrm{m}}^{2} / 2
$$

The relativistically correct Doppler equation for a source moving radially away with velocity $v=\beta c$ is

$$
\frac{v}{v_{0}}=\left(\frac{1-\beta}{1+\beta}\right)^{1 / 2} .
$$

After the observed frequency had been corrected for the gravitational time dilation and transverse Doppler shift, we used 
Table 3

Sample Data for NGC 6264

\begin{tabular}{ccccccc}
\hline \hline $\begin{array}{c}V_{\text {op }^{\mathrm{a}}} \\
\left(\mathrm{km} \mathrm{s}^{-1}\right)\end{array}$ & $\begin{array}{c}\text { R.A. }^{\mathrm{b}} \\
(\mathrm{mas})\end{array}$ & $\begin{array}{c}\delta \text { R.A. }^{\mathrm{b}} \\
(\mathrm{mas})\end{array}$ & $\begin{array}{c}\text { Decl. }^{\mathrm{b}} \\
(\mathrm{mas})\end{array}$ & $\begin{array}{c}\delta \text { Decl. } \\
(\mathrm{mas})\end{array}$ & $\begin{array}{c}\mathrm{F}_{\nu}{ }^{\mathrm{c}} \\
\left(\mathrm{mJy} \mathrm{B}^{-1}\right)\end{array}$ & $\begin{array}{c}\sigma_{F}{ }^{\mathrm{c}} \\
\left(\mathrm{mJy} \mathrm{B}^{-1}\right)\end{array}$ \\
\hline 10918.33 & 0.399 & 0.008 & -0.021 & 0.018 & 6.0 & 0.3 \\
10914.71 & 0.407 & 0.010 & -0.001 & 0.021 & 4.8 & 0.3 \\
10911.09 & 0.401 & 0.005 & 0.000 & 0.011 & 10.2 & 0.4 \\
10907.47 & 0.397 & 0.013 & 0.041 & 0.029 & 3.7 & 0.3 \\
10903.85 & 0.395 & 0.011 & -0.050 & 0.027 & 3.8 & 0.4 \\
10900.23 & 0.388 & 0.009 & 0.044 & 0.022 & 4.6 & 0.3 \\
10885.74 & 0.369 & 0.019 & 0.053 & 0.038 & 2.6 & 0.4 \\
10871.26 & 0.400 & 0.018 & 0.061 & 0.037 & 2.9 & 0.3 \\
10856.77 & 0.467 & 0.011 & -0.038 & 0.029 & 3.8 & 0.3 \\
10853.15 & 0.484 & 0.005 & -0.038 & 0.011 & 9.7 & 0.4 \\
10849.53 & 0.490 & 0.005 & -0.006 & 0.011 & 9.4 & 0.3 \\
10845.91 & 0.492 & 0.002 & -0.016 & 0.006 & 18.3 & 0.4 \\
10842.29 & 0.494 & 0.002 & -0.005 & 0.005 & 24.4 & 0.4 \\
10838.67 & 0.505 & 0.004 & -0.010 & 0.009 & 11.7 & 0.4 \\
10835.05 & 0.543 & 0.012 & -0.094 & 0.032 & 3.5 & 0.4 \\
10831.43 & 0.542 & 0.017 & -0.039 & 0.031 & 3.1 & 0.3 \\
10827.81 & 0.518 & 0.012 & -0.101 & 0.027 & 3.9 & 0.3 \\
10824.19 & 0.533 & 0.007 & -0.040 & 0.016 & 6.9 & 0.4 \\
10820.56 & 0.493 & 0.012 & -0.041 & 0.026 & 3.8 & 0.3 \\
\hline
\end{tabular}

Notes. Sample of data for NGC 6264.

a Velocity referenced to the LSR and using the optical definition (no relativistic corrections)

b East-west and north-south position offsets and uncertainties measured relative to the average position of the systemic masers in the VLBI map (Figure 2). Position uncertainties reflect fitted random errors only. In NGC 1194 and NGC 2960, there may be additional uncertainties caused by the poorer tropospheric delay calibrations due to their low declinations (low elevations during the observations).

${ }^{c}$ Fitted peak intensity and its uncertainty in mJy beam ${ }^{-1}$.

(This table is available in its entirety in a machine-readable form in the online journal. A portion is shown here for guidance regarding its form and content.)

Equation (5) to convert the corrected frequency to its relativistically correct radial velocity $v=\beta c$. For the megamaser disks, typically $\beta_{\mathrm{m}}<0.003$. So, for galaxies in the Hubble flow $(z>0.01)$, the general relativistic corrections are smaller than the special relativistic corrections. For example, for masers in UGC 3789, which has an optical-LSR recession velocity of $3262 \mathrm{~km} \mathrm{~s}^{-1}$, the special relativistic corrections range from 10 to $26 \mathrm{~km} \mathrm{~s}^{-1}$ whereas the general relativistic corrections range from 0 to $\sim 2 \mathrm{~km} \mathrm{~s}^{-1}$.

Finally, when fitting the rotation curves, we used the relativistic formula for the addition of velocities to decompose the observed $\beta$ value of each maser spot into a common $\beta_{\mathrm{g}}$ associated with the radial velocity of the center of each megamaser disk and an individual $\beta_{\mathrm{m}}$ associated with orbital motion of a specific maser spot. The redshifted maser spots have $\beta_{\mathrm{m}}>0$ and blueshifted maser spots have $\beta_{\mathrm{m}}<0$.

$$
\beta=\frac{\beta_{\mathrm{g}}+\beta_{\mathrm{m}}}{1+\beta_{\mathrm{g}} \beta_{\mathrm{m}}} .
$$

\section{RESULTS}

\subsection{VLBI Images, Rotation Curves, and BH Masses}

Figure 1 shows the GBT single-dish spectra for all megamaser galaxies presented here except for UGC 3789, which can be found in Reid et al. (2009a). Figures 2, 3, and 4 show the VLBI maps and the position-velocity $(P-V)$ diagrams along with the fitted rotation curves of the maser spectral components (spots) in UGC 3789, NGC 1194, NGC 2273, NGC 2960 (Mrk 1419), NGC 4388, NGC 6264, and NGC 6323. Reid et al. (2009a) published the UGC 3789 VLBI map and the rotation curve, and we performed a new analysis of the BH mass for this galaxy based on those data. We show the VLBI map and rotation curve for this galaxy again for direct comparison with the other six megamaser disks. The data points in the VLBI maps and rotation curves are color-coded to indicate redshifted, blueshifted, and systemic masers, where the "systemic" masers refer to the maser spectral components having velocities close to the systemic velocity of the galaxy. Except for NGC 4388, the maser spot distributions are plotted relative to the average position of the systemic masers. Systemic masers are not detected in NGC 4388, so we plotted its maser distribution relative to the dynamical center determined from fitting the data in the $P-V$ diagram with a Keplerian rotation curve.

To estimate the inclination and dynamical center (i.e., the position of the $\mathrm{BH}$ ) of each disk, we rotated the coordinate system to make the disk horizontal and used the fitted horizontal line that passes through the high-velocity masers as the zero point of the $y$-coordinate of the dynamical center (see Figures 3 and 4). The zero point of the $x$-coordinate is defined to be the unweighted average $\theta_{\mathrm{x}}$ of the systemic masers.

We assumed that the systemic masers have about the same orbital radii as the high-velocity masers to estimate the maser disk inclination $\cos ^{-1}\left(\left\langle\theta_{\mathrm{y}}^{\text {(sys) }}\right\rangle / \theta_{\mathrm{r}}\right)$, where $\left\langle\theta_{\mathrm{y}}^{\text {(sys) }}\right\rangle$ is the average $\theta_{\mathrm{y}}$ position of the systemic masers and $\theta_{\mathrm{r}}$ is the orbital radius of the systemic masers. In principle, one can determine $\theta_{\mathrm{r}}$ exactly only when good rotation curves for both systemic and high-velocity masers can be obtained, and precise acceleration measurements for the systemic masers are available. Among our data we only have such information for UGC 3789 at this point (Braatz et al. $2010)$, and so we use $\left\langle\theta_{\mathrm{x}}\right\rangle$ of high-velocity masers as an estimate of $\theta_{\mathrm{r}}$. We note that all our megamaser disks with systemic masers detected have inclinations larger than $80^{\circ}$, so assuming the disk is exactly edge-on will only cause errors less than $1 \%$ in the derived BH masses. In NGC 4388 we could not measure a disk inclination, but even if the disk were $20^{\circ}$ from edge-on, the contribution to the error in the $\mathrm{BH}$ mass would be only $12 \%$.

We determined the rotation curve for each megamaser disk as a function of the "impact parameter" defined as the projected radial offset $\theta=\left(\theta_{\mathrm{x}}^{2}+\theta_{\mathrm{y}}^{2}\right)^{1 / 2}$ of the maser spots so that we can account for the warped structures in some megamaser disks. We then performed a nonlinear least-squares fit of a Keplerian rotation curve to the $P-V$ diagram with the assumption that the high-velocity masers lie exactly on the mid-line of the disk. In addition, the systemic velocity of each galaxy was fitted as a free parameter, and we report the best fits of the systemic velocities of our megamaser galaxies in Table 1.

The fitted Keplerian rotation curves can be expressed with the following form:

$$
\left|v_{\mathrm{K}}\right|=v_{1}\left(\frac{\theta}{1 \mathrm{mas}}\right)^{-1 / 2}
$$

where $\left|v_{\mathrm{K}}\right|$ is the orbital velocity (after relativistic corrections) of the high-velocity masers and $v_{1}$ is the orbital velocity at a radius 1 mas from the dynamical center. The BH mass is

$$
M_{\bullet}=\left(\frac{\left|v_{\mathrm{K}}\right|^{2} \theta}{\mathrm{G}}\right) D_{\mathrm{A}}=\left(\frac{\pi v_{1}^{2}}{6.48 \times 10^{8} \mathrm{G}}\right) D_{\mathrm{A}},
$$

where $D_{A}$ is the angular diameter distance to the galaxy. We show all the measured BH masses in Table 4. 

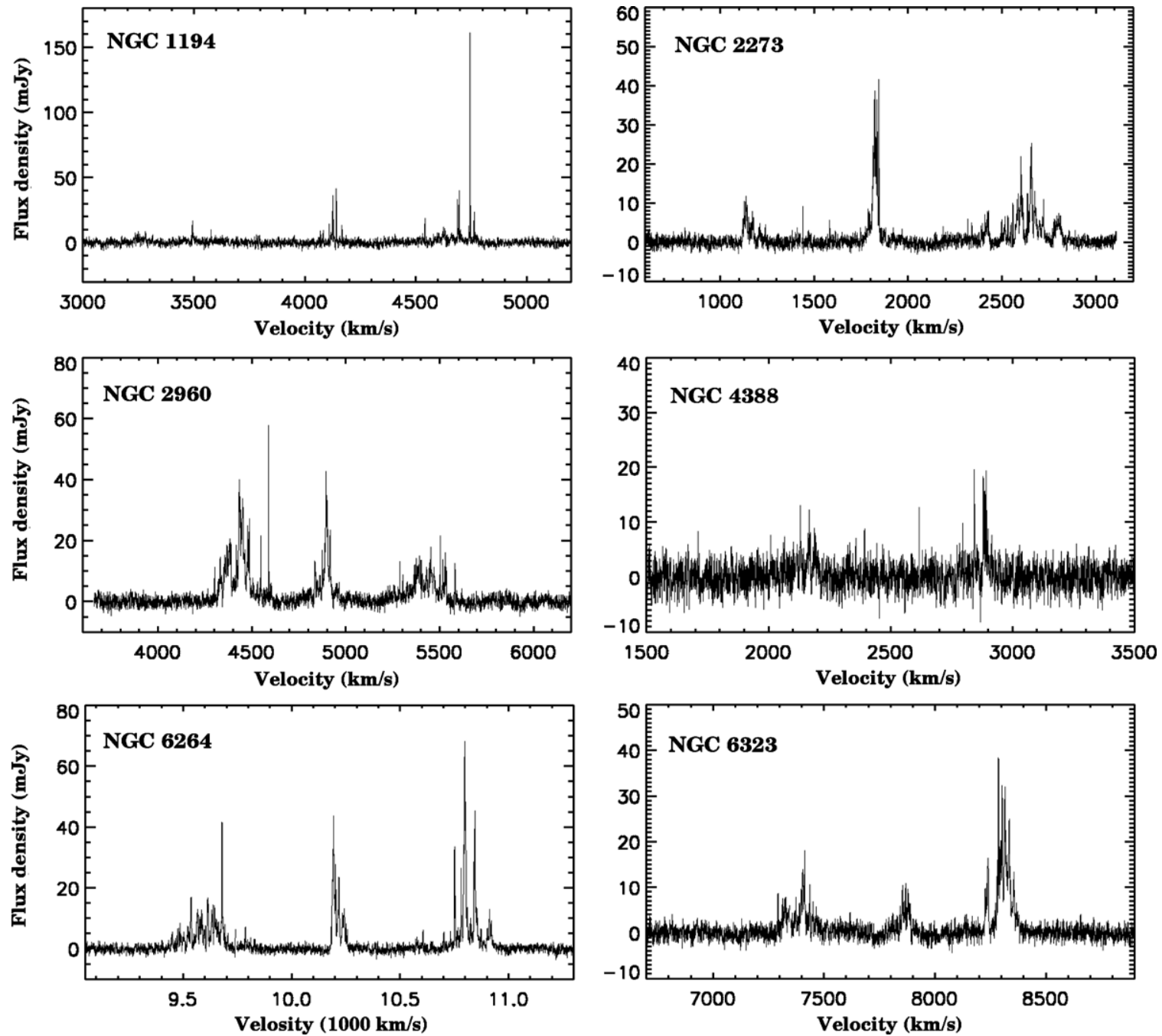

Figure 1. Characteristic $\mathrm{H}_{2} \mathrm{O}$ maser spectra. The $x$-axis shows LSR velocities based on optical definition. Flux densities of masers can vary significantly, so the spectra shown here are just representative for particular epochs: 2008 January 13 for NGC 1194; 2009 February 21 for NGC 2273; 2009 April 2 for NGC 2960 (Mrk 1419); 2005 November 30 for NGC 4388; 2009 March 31 for NGC 6264; and 2000 April 6 for NGC 6323.

Table 4

The BH Masses and Basic Properties of the Maser Disks

\begin{tabular}{|c|c|c|c|c|c|c|c|c|c|c|}
\hline Name & $\begin{array}{c}\text { Dist. } \\
(\mathrm{Mpc})\end{array}$ & $\begin{array}{l}\text { BH mass } \\
\left(10^{7} M_{\odot}\right)\end{array}$ & $\begin{array}{c}\text { Disk Size } \\
(\mathrm{pc})\end{array}$ & $\begin{array}{l}\text { P.A. } \\
\left({ }^{\circ}\right)\end{array}$ & $\begin{array}{c}\text { Incl. } \\
\left({ }^{\circ}\right)\end{array}$ & $\begin{array}{c}r_{\mathrm{c}} \\
(\mathrm{pc})\end{array}$ & $\begin{array}{c}\rho_{0} \\
\left(M_{\odot} \mathrm{pc}^{-3}\right)\end{array}$ & $\begin{array}{l}m_{\max } \\
\left(M_{\odot}\right)\end{array}$ & $\begin{array}{l}T_{\text {age }} \\
(\mathrm{yr})\end{array}$ & $\begin{array}{c}R_{\text {inf }} \\
(\operatorname{arcsec})\end{array}$ \\
\hline NGC 1194 & 53.2 & $6.5 \pm 0.3$ & $0.54-1.33$ & 157 & 85 & 0.260 & $1.2 \times 10^{9}$ & 13 & $>1.0 \times 10^{10}$ & 0.033 \\
\hline NGC 2273 & 25.7 & $0.75 \pm 0.04$ & $0.028-0.084$ & 153 & 84 & 0.015 & $6.1 \times 10^{11}$ & 0.05 & $<2.2 \times 10^{6}$ & 0.010 \\
\hline UGC 3789 & 46.4 & $1.04 \pm 0.05$ & $0.084-0.30$ & 41 & $>88$ & 0.022 & $2.3 \times 10^{11}$ & 0.1 & $<1.8 \times 10^{7}$ & 0.010 \\
\hline NGC 2960 & 72.2 & $1.16 \pm 0.05$ & $0.13-0.37$ & -131 & 89 & 0.056 & $1.7 \times 10^{10}$ & 0.48 & $<1.0 \times 10^{9}$ & 0.005 \\
\hline NGC 4388 & 19.0 & $0.84 \pm 0.02$ & $0.24-0.29$ & 107 & - & 0.090 & $3.3 \times 10^{9}$ & 0.9 & $<6.6 \times 10^{9}$ & 0.034 \\
\hline NGC 6264 & 139.4 & $2.91 \pm 0.04$ & $0.24-0.80$ & -85 & 90 & 0.085 & $1.2 \times 10^{10}$ & 1.4 & $>1.0 \times 10^{10}$ & 0.012 \\
\hline NGC 6323 & 106.0 & $0.94 \pm 0.01$ & $0.13-0.30$ & 10 & 89 & 0.046 & $2.3 \times 10^{10}$ & 0.3 & $<4.7 \times 10^{8}$ & 0.003 \\
\hline
\end{tabular}

Notes. Column 1: galaxy name. Column 2: the Hubble flow distances we adopt from NED. Column 3: BH masses measured in our project. The mass uncertainty here only includes errors caused by source position uncertainty and from fitting a Keplerian rotation curve for a given distance. Except for NGC 4388 , the actual BH mass uncertainty is dominated by the error of the latest $H_{0}$ measurement $(\sim 6 \%)$. NGC 4388 has a larger BH mass error $(11 \%)$ which is limited by the uncertainty of the Tully-Fisher distance determination(see the explanation in Section 3.2). Column 4: sizes of the inner and outer edge of the maser disks. Column 5: position angle (P.A.) of the disk plane measured east of north. P.A. equals zero when the blueshifted side of the disk plane has zero east offset and positive north offset. Column 6: inclination of the maser disk. Note that the inclination of NGC 4388 is unconstrained because we did not detect systemic masers. Column 7: the core radius of the Plummer cluster in parsecs. For NGC 1194, NGC 2273, NGC 2960, and NGC 4388, the core radii are derived from Equation (12). For UGC 3789, NGC 6264, and NGC 6323, the radii are derived from the Plummer rotation curve fitting. Column 8: the central mass density of the Plummer cluster $\rho_{0}=3 M_{\infty} / 4 \pi r_{c}^{3}$. Here, $M_{\infty}$ is obtained from the Plummer rotation curve fitting. Column 9: the maximum stellar mass of the Plummer cluster below which the cluster will not evaporate in less than 10 Gyr. In all cases except NGC 1194, a cluster of neutron stars can be directly ruled out because $m_{\max }$ is less than $\approx 1.4 M_{\odot}$. Column 10 : lifetime ( $T_{\text {age }}$ ) of a cluster. The values shown here are limited by the collision timescale, which is the maximum lifetime of the cluster composed of either main-sequence stars, brown dwarfs, white dwarfs, or neutrons stars. Column 11: the radius of the gravitational sphere of influence for the maser BHs in arcsec. We calculate $R_{\text {inf }}$ using Equation (1) of Barth et al. (2004) with the bulge velocity dispersion measurements from Greene et al. (2010). 

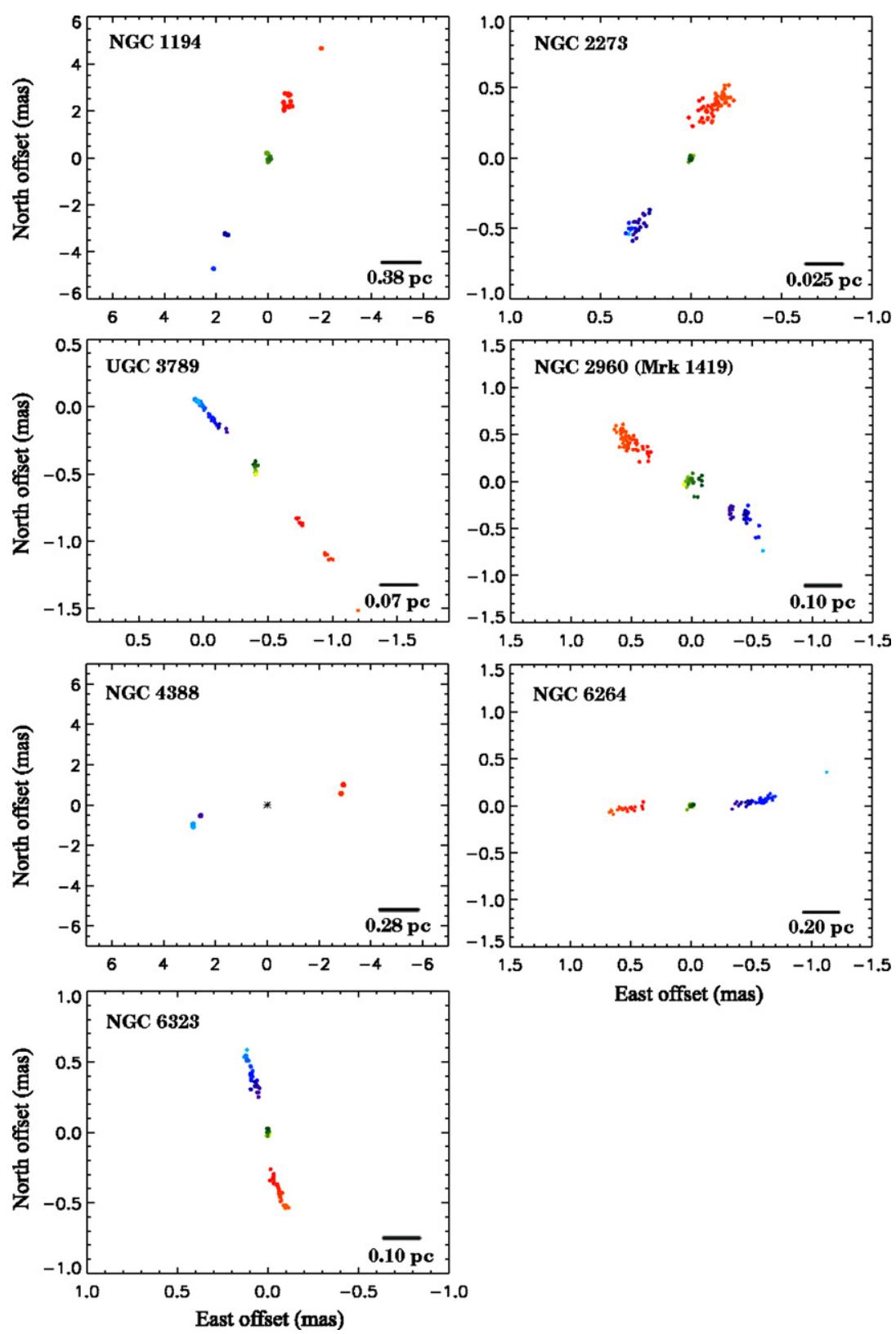

Figure 2. VLBI maps for the seven $22 \mathrm{GHz} \mathrm{H}_{2} \mathrm{O}$ masers megamasers analyzed. The maps are color-coded to indicate redshifted, blueshifted, and systemic masers, where the "systemic" masers refer to the maser components having recessional velocities close to the systemic velocity of the galaxy. Except NGC 4388, maser distributions are plotted relative to the average position of the systemic masers. For NGC 4388, in which the systemic masers are not detected, we plot the maser distribution relative to the dynamical center determined by fitting the high-velocity features with a Keplerian rotation curve.

\subsection{The Error Budget for the BH Mass}

There are primarily three sources of error for our BH mass calculation, and the largest comes from the distance uncertainty. Except for NGC 4388, we used the Hubble flow distances (relative to the $3 \mathrm{~K}$ cosmic microwave background) from the NASA/IPAC Extragalactic Database (NED) for a cosmological model with $H_{0}=73 \mathrm{~km} \mathrm{~s}^{-1} \mathrm{Mpc}^{-1}, \Omega_{\text {matter }}=0.27$, and $\Omega_{\Lambda}=$ 0.73 . These distances have an error about $6 \%$ (assuming the error from the peculiar velocity of the galaxy is negligible), caused by the uncertainty of the latest Hubble constant measurements (Freedman \& Madore 2010; Riess et al. 2009). Since NGC 4388 is in the Virgo cluster and has a large peculiar velocity, the Hubble distance can have significant uncertainty. Therefore, we adopted its Tully-Fisher distance of $19 \mathrm{Mpc}$, which has an error about 11\% (Russell 2002).
The second source of BH mass uncertainty comes from fitting the rotation curves of the masers. In our Keplerian fitting, the rms $\sigma_{\theta}$ of the observed position offsets from Keplerian rotation is usually larger than the rms position uncertainties of the data by a factor of 1.5-4. This extra scatter can lead to a systematically different position of the dynamical center, and hence a slightly different $\mathrm{BH}$ mass if we allow dynamical center position to be a free parameter in the fit. This excess may indicate that we underestimated our observational errors or it may indicate genuine deviations from our simple model, possibly caused by peculiar motions of the masers (e.g., perturbations from spiral density waves) or high-velocity masers not lying precisely on the mid-line of the disk (Humphreys et al. 2008). If the latter is the main cause of deviation, we estimate that the majority of the maser spots fall within $\approx 7^{\circ}-10^{\circ}$ from the midline of the disk, depending on the galaxy. 

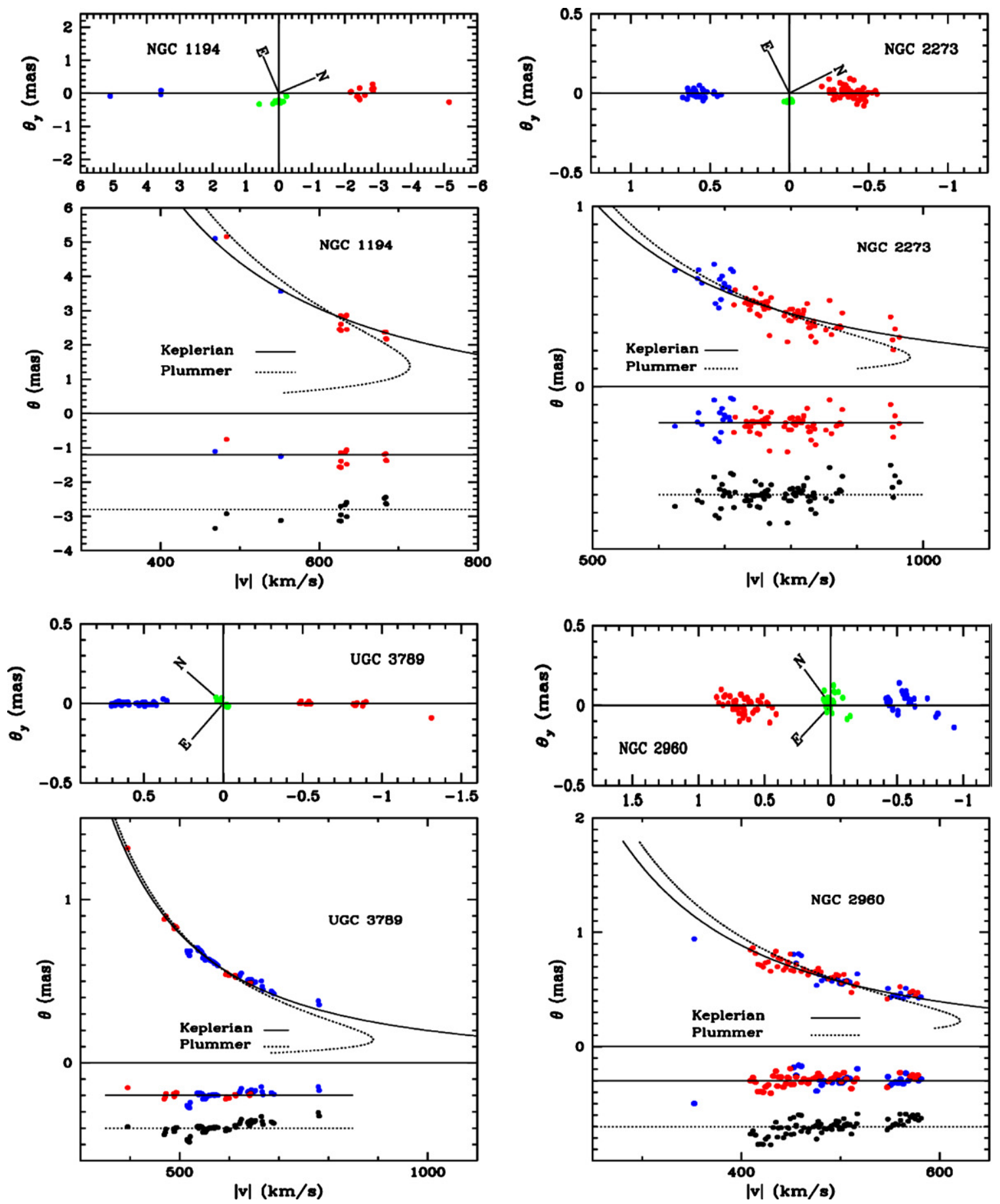

Figure 3. Maser distributions (top panels) and rotation curves (bottom panels) for NGC 1194, NGC 2273, UGC 3789, and NGC 2960. The maser distribution has been rotated to horizontal to show the scatter in the maser positions and the offset of the systemic masers from the plane defined by high-velocity masers more clearly. The coordinate system is chosen to place the centroid of the high-velocity maser disk (blue and red points) at $\theta_{\mathrm{y}}=0$ and the centroid of the systemic masers (green points) at $\theta_{\mathrm{x}}=0$. The axes for the maps show relative position in milliarcseconds, and north $(\mathrm{N})$ and east (E) are indicated by directional arrows on each map. The bottom panel for each galaxy shows the rotation curves of the redshifted and blueshifted masers (red and blue points on the curves) plotted with the best-fit Keplerian (solid curve) and Plummer (dotted curve) rotation curves. The velocities shown in the figure are the LSR velocities after the special and general relativistic corrections. The residuals (data minus Keplerian curve in red and blue; data minus Plummer curve in black) are in the bottom part of each figure. Note that we plot the rotation curve with the impact parameter $\theta$ (mas) as the ordinate and rotation speed $|v|\left(\mathrm{km} \mathrm{s}^{-1}\right)$ as the abscissa for the convenience of fitting.

In addition to the deviations of the masers from the midline of the maser disk, there is also an error in the $\mathrm{BH}$ mass from the uncertainty of the position of the $\mathrm{BH}$ in the fitting. To estimate this error, we relax our assumption on the position of the $\mathrm{BH}$, and allow it to be a free parameter in the fitting. However, since the recession velocity of the galaxy $\left(v_{0}\right)$ and the position of the $\mathrm{BH}$ are correlated in this case, we impose a constraint on the possible positions of the $\mathrm{BH}$ such that the fitted $v_{0}$ does not exceed $v_{0}$ determined from other methods (e.g., H I measurements) beyond their error bars. Including all possible errors mentioned above, we estimate the total fitting error in $\mathrm{BH}$ mass to be $1 \%-5 \%$ depending on the galaxy.

The third source of error is from the absolute position errors of our megamasers. Source position errors introduce an extra phase 

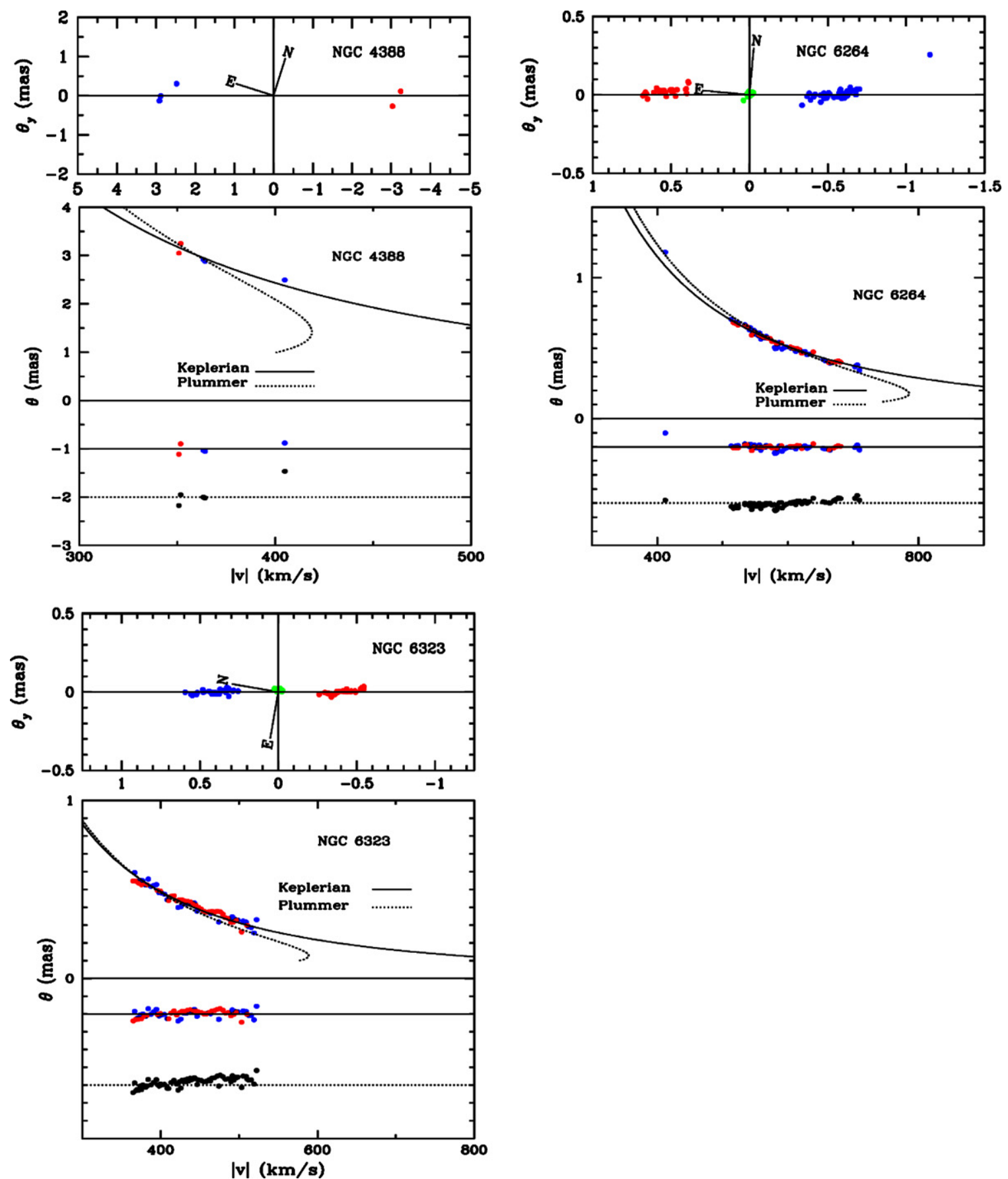

Figure 4. Maser distributions (top panel) and rotation curves (bottom panel) for NGC 4388, NGC 6264, and NGC 6323. Please refer to the caption of Figure 3 for the description of this figure.

difference between maser spots having different frequencies (velocities). The relative position errors scale with velocity offset from the reference maser feature (Argon et al. 2007; Greenhill et al. 1993), which may differ from source to source. Since the BH mass is proportional to the size of the maser orbits, relative position shifts among masers introduce errors in the BH mass measurements. Among our megamasers, UGC 3789, NGC 2960, and NGC 2273 have the largest absolute position errors (10 mas; see Note $h$ in Table 1). The resultant BH mass errors are $3 \%-5 \%$, whereas this error is smaller than $0.3 \%$ in the other megamaser galaxies. Please note that the error for the $\mathrm{BH}$ mass discussed in the following subsections does not include the distance uncertainty. Only source position errors and errors from Keplerian fitting are evaluated, which is the best approach for comparing our results with $\mathrm{BH}$ masses derived from other techniques as the same distance can be used for comparison. As the distance uncertainties shrink in the future, so will the uncertainties in our BH masses.

\subsection{Notes on Individual Galaxies \\ 3.3.1. NGC 1194}

NGC 1194 hosts a Seyfert 1.9 nucleus and has a distance of $\approx 52 \mathrm{Mpc}$. The position angle of the maser disk (Figure 2 ) is $157^{\circ}$ and the inclination is $\approx 85^{\circ}$. NGC 1194 has the largest maser disk among the megamasers presented here, with an inner and 
outer radius of 0.54 and $1.33 \mathrm{pc}$, respectively. The blueshifted and redshifted masers do not appear to fall exactly on a straight line on the sky, but they are consistent with a slightly bent thin disk. The rotation curve (Figure 3 ) is consistent with Keplerian rotation, and the measured $\mathrm{BH}$ mass is $(6.5 \pm 0.3) \times 10^{7} \mathrm{M}_{\odot}$, the largest among the $\mathrm{BH}$ masses studied here.

\subsubsection{NGC 2273}

NGC 2273 is a Seyfert 2 galaxy at a distance of $\approx 26 \mathrm{Mpc}$. The position angle of the maser disk (Figure 2 ) is $153^{\circ}$ with an inclination of $\approx 84^{\circ}$. The inner and outer radii of the disk are 0.028 and $0.084 \mathrm{pc}$, respectively. The maser distribution shows a hint of a warped disk. The rotation curve (Figure 3 ) is also consistent with Keplerian rotation to within the errors. The $\mathrm{BH}$ mass we obtain is $(7.5 \pm 0.4) \times 10^{6} M_{\odot}$.

\subsubsection{UGC 3789}

UGC 3789 is a Seyfert 2 galaxy at a distance of $\approx 50 \mathrm{Mpc}$ (Reid et al. 2009a; Braatz et al. 2010). Its edge-on maser disk lies at position angle $41^{\circ}$ east of north (Figure 2). Although the high-velocity masers appear to trace a flat disk, the misalignment of the systemic masers with respect to the disk and the fact that the systemic masers have at least two distinct centripetal accelerations (Braatz et al. 2010) suggest that the disk is probably warped along the line of sight. The inner and outer radii of the disk are 0.084 and $0.30 \mathrm{pc}$, respectively. Fitting the $P-V$ diagram (Figure 3 ) with a Keplerian rotation curve gives a BH mass of $(1.04 \pm 0.05) \times 10^{7} M_{\odot}$.

\subsubsection{NGC 2960 (Mrk 1419)}

NGC 2960 hosts a LINER nucleus at a distance of $\approx 71 \mathrm{Mpc}$. The position angle of the maser disk (Figure 2) is $-131^{\circ}$ and the inclination is $\approx 89^{\circ}$. The outer parts of the disk show some warping. The inner and outer radii of the disk are 0.13 and 0.37 pc, respectively. The rms scatter of the high-velocity masers normal to the disk is $\sigma_{\perp}=71 \mu \mathrm{as}$, which is 1.6 times larger than the rms uncertainty of the data, so it is likely that either we underestimated the observational uncertainties because of larger tropospheric delay errors for low declination sources, or the thickness of the disk may not be negligible in this megamaser. In this galaxy, we measure a BH mass of $(1.16 \pm 0.05) \times 10^{7} M_{\odot}$.

\subsection{5. $N G C 4388$}

NGC 4388 is in the Virgo cluster and we adopted a Tully-Fisher distance of $19 \mathrm{Mpc}$; it is the nearest of the seven galaxies presented here. It hosts a Seyfert 2 nucleus and the maser disk (Figure 2) has a position angle of $107^{\circ}$. We cannot measure the inclination directly because no systemic masers were detected. As with the other megamasers, we assumed a flat, edge-on disk.

Since no systemic masers were detected, we determined the position of the dynamical center by allowing it to be a free parameter when fitting the high-velocity masers with a Keplerian rotation curve (Figure 4). In addition, we fixed the systemic velocity of the galaxy using an $\mathrm{H}_{\mathrm{I}}$ measurement $(\mathrm{Lu}$ et al. 2003). The resulting BH mass is $(8.5 \pm 0.2) \times 10^{6} M_{\odot}$. With only five maser spots mapped, there are not sufficient data to demonstrate Keplerian rotation or even to show that the masers actually lie on a disk. We argue that it is likely they do because the radio continuum jet shown by Kukula et al. (1995) is nearly perpendicular to the line joining the blueshifted and redshifted masers. However, because of these uncertainties, the
BH mass for NGC 4388 should be used with some caution until better data are obtained.

\subsection{6. $N G C 6264$}

At $\approx 136$ Mpc, NGC 6264 (Figure 2) is the most distant object in our current sample. The disk is slightly warped and appears to have some thickness, but given the uncertainties in the position measurement, our observation is consistent with a thin disk. The disk has a position angle of $-85^{\circ}$ and an inclination of $\approx 90^{\circ}$. The inner and outer radii of the disk are 0.24 and $0.80 \mathrm{pc}$, respectively. In the $P-V$ diagram (Figure 4), the high-velocity masers beautifully trace the Keplerian rotation curve, and we obtain a BH mass of $(2.91 \pm 0.04) \times 10^{7} M_{\odot}$.

\subsection{7. $N G C 6323$}

NGC 6323 is a Seyfert 2 galaxy at a distance of $\approx 105 \mathrm{Mpc}$. The VLBI image (Figure 2) shows a remarkably thin disk at a position angle of $10^{\circ}$ and inclination of $\approx 89^{\circ}$. The disk is apparently warped, and the inner and outer radii are 0.13 and $0.3 \mathrm{pc}$, respectively. The rotation curve of the high-velocity masers (Figure 4) is Keplerian, and we obtain a BH mass of $(9.4 \pm 0.1) \times 10^{6} M_{\odot}$.

\subsection{Search for Continuum Emission}

We searched for continuum emission from the vicinity of the supermassive BH (i.e., near the systemic masers) in our megamaser galaxies by averaging the line-free spectral channels in our data and imaging with natural weighting to maximize the detection sensitivity. We detected no continuum emission in all megamaser galaxies presented in this paper. The channels averaged, the center velocities of the bands used for averaging, and the continuum upper limits are listed in Table 5.

\section{A SUPERMASSIVE BLACK HOLE OR A CENTRAL CLUSTER OF STARS OR STELLAR REMNANTS ?}

The near-Keplerian shapes of the rotation curves of many circumnuclear megamaser disks allows one to determine the enclosed (spherical) mass within the innermost radius of the disk. While the enclosed masses of the megamaser disks are very likely dominated by supermassive BHs, as we assumed in the previous sections, it is still important to find ways to justify this assumption. The question of whether the enclosed mass can be attributed to a point mass or a compact cluster of stars or stellar remnants has been addressed by Maoz (1995) $\&$ Maoz (1998). The main argument is that if the lifetime of a central cluster, limited by evaporation or collision timescales, is significantly shorter than the age of its host galaxy, then it is unlikely to persist, and a central supermassive BH would be required to account for the enclosed mass. Here, the evaporation timescale $t_{\text {evap }} \approx 136 t_{\text {relax }}$ ( $t_{\text {relax }}$ is the half-mass relaxation timescale; Binney \& Tremaine 2008) is considered to be the upper limit of the lifetime of any bound stellar system, whereas the collision timescale is the characteristic timescale that a star suffers a physical collision (i.e., an inelastic encounter; Binney \& Tremaine 2008).

To estimate the lifetime of the central cluster, we follow Maoz (1995) and assume that the central cluster has the Plummer density distribution (Plummer 1915):

$$
\rho(r)=\rho_{0}\left(1+\frac{r^{2}}{r_{\mathrm{c}}^{2}}\right)^{-5 / 2},
$$


Table 5

Upper Limit on Continuum Emission from Megamaser Galaxies

\begin{tabular}{lcccc}
\hline \hline Galaxy & $\begin{array}{c}V_{\text {Center }} \\
\left(\mathrm{km} \mathrm{s}^{-1}\right)\end{array}$ & $\begin{array}{c}V_{\text {Range }} \\
\left(\mathrm{km} \mathrm{s}^{-1}\right)\end{array}$ & $\begin{array}{c}I_{2 \sigma} \\
(\mathrm{mJy})\end{array}$ & Project Code \\
\hline NGC 1194 & 3568 & $3489-3660$ & $<0.34$ & BB242B \\
NGC 2273 & 2800 & $2868-2909$ & $<0.18$ & BB261B \\
NGC 2960 & 5315 & $5296-5354$ & $<0.14$ & BB261C, BB261D \\
NGC 4388 & 2600 & $2881-2966$ & $<0.42$ & BB184C \\
NGC 6264 & 9710 & $9726-9776$ & $<0.16$ & BB261F, BB261H \\
NGC 6323 & 8100,7650 & $8005-8200,7556-7750$ & $<0.08$ & BB242M, BB242R, BB242S, BB242T
\end{tabular}

Notes. Column 1: name of the galaxy. Column 2: the central optical-LSR velocity of the bands used to search for continuum emission. Column 3: the velocity range corresponding to the selected channels. Note that the channels selected are chosen to be free of any maser lines except for NGC 2960. For this galaxy, we do not have line-free channels, and we averaged the bands centered at the systemic velocity of the galaxy and searched for continuum emission offset from the systemic maser emission. Column 4 : the $2 \sigma$ detection limit of the continuum emission. Column 5: the data used for continuum detection.

where $\rho_{0}$ is the central density and $r_{\mathrm{c}}$ is the core radius. (The reason for choosing the Plummer distribution is described in Section 2.1 of Maoz 1998.)

We constrain $\rho_{0}$ and $r_{\mathrm{c}}$ by fitting the $P-V$ diagram of the megamaser disk with the rotation curve of a Plummer cluster:

$$
v_{\mathrm{P}}=\left[\frac{G M_{\infty} r^{2}}{\left(r_{\mathrm{c}}^{2}+r^{2}\right)^{3 / 2}}\right]^{1 / 2}
$$

where $M_{\infty}=4 \pi \rho_{0} r_{c}^{3} / 3$ is the total mass of the cluster. Here, $M_{\infty}$ and $r_{\mathrm{c}}$ are fitted as free parameters. From these two parameters, we calculated $\rho_{0}=3 M_{\infty} / 4 \pi r_{c}^{3}$. In all cases $M_{\infty}$ is very close to the "enclosed" mass measured from the Keplerian rotation curve fit in Section 3, and the differences are less than 4\%-18\%. In Figures 3 and 4, we show the fitted Plummer rotation curves along with the fitted Keplerian rotation curves.

Rather than using the method described above to constrain the core radius $r_{c}$, in some cases we could apply another approach that places even tighter constraints. We note that the Plummer rotation curve does not decrease monotonically with radius; instead the rotation curve turns over at a maximum rotation speed $v_{\max }$ :

$$
v_{\max }=\left(\frac{2 G M_{\infty}}{3^{3 / 2} r_{\mathrm{c}}}\right)^{1 / 2}
$$

Having a maximum rotation velocity is not unique to a Plummer cluster. It is a general feature for clusters having the same form of density profile with the exponent smaller than $-3 / 2$.

The core radius $r_{\mathrm{c}}$ of a Plummer sphere having maximum rotation speed $v_{\max }$ is

$$
r_{\mathrm{c}}^{(\max )}=\frac{2 G M_{\infty}}{3^{3 / 2} v_{\max }^{2}}
$$

We used Equation (12) to estimate $r_{c}$ for the megamaser disks in NGC 1194, NGC 2273, NGC 2960, and NGC 4388. In these cases, we do not have very well-sampled or high quality rotation curves (see Figures 3 and 4), so using $r_{\mathrm{c}}^{(\max )}$ from Equation (12) actually sets a tighter constraint on the core radius of the cluster than using rotation-curve fitting. For these four cases, we use the highest observed velocity in the PV diagram as an estimate (lower limit) of $v_{\max }$ and use it to calculate $r_{c}^{(\max )}$ with $M_{\infty}$ from the Plummer rotation curve fitting from Equation (10). For UGC 3789, NGC 6323, and NGC 6264, we used $r_{c}$ from rotation curve fitting because the qualities of the rotation curves are good, and they give tighter constraints on $r_{\mathrm{c}}$. In Table 4, we give the Plummer model parameters for all of our megamaser disks.

We constrained the lifetime of the Plummer cluster $T_{\text {age }}$ by first requiring the cluster not evaporate in a timescale less than the age of its host galaxy ( $\geqslant 10 \mathrm{Gyr}$ if the galaxy has formed before $z=2$ ). This requirement sets an upper limit to the mass of the constituent stars of the cluster because evaporation is unimportant so long as the mass of its stars satisfies the following equation:

$$
\left(\frac{m_{\star}}{M_{\odot}}\right) \lesssim\left[\ln \left(\lambda M_{\infty} / m_{\star}\right)\right]^{-1}\left(\frac{r_{\mathrm{h}}}{0.01 \mathrm{pc}}\right)^{3 / 2}\left(\frac{M_{\infty}}{10^{8} M_{\odot}}\right)^{1 / 2}
$$

where $m_{\star}$ is the mass of each star, $\lambda \approx 0.1$ (Binney \& Tremaine $2008)$, and $r_{\mathrm{h}}$ is the radius of half total mass $\left(r_{\mathrm{h}} \approx 1.305 r_{\mathrm{c}}\right)$. We call the maximum $m_{\star}$ that satisfies the above equation $m_{\max }$ (Table 4) and used it to calculate the collision timescale of the Plummer cluster with the Plummer model parameters:

$$
t_{\text {coll }}=\left[16 \sqrt{\pi} n_{\star} \sigma_{\star} r_{\star}^{2}\left(1+\frac{G m_{\text {max }}}{2 \sigma_{\star}^{2} r_{\star}}\right)\right]^{-1},
$$

where $n_{\star}=\rho_{0} / m_{\max }$ is the number density of stars, $\sigma_{\star}$ is the rms velocity dispersion of the stars, and $r_{\star}$ is the stellar radius (Maoz 1998; Binney \& Tremaine 2008). If $t_{\text {coll }}<10$ Gyr, then $T_{\text {age }}$ is constrained by $t_{\text {coll }}$ and we can rule out the Plummer cluster as an alternative to the BH. If $t_{\text {coll }} \geqslant 10 \mathrm{Gyr}$, we cannot rule out a cluster whether $T_{\text {age }}$ is dominated by evaporation or collision.

For UGC 3789 we obtained an upper limit $m_{\max } \approx 0.10 M_{\odot}$ to the mass of individual stars and a lower limit $N \gtrsim 1.0 \times 10^{8}$ to the number of stars in the cluster. The mass limit directly rules out neutron stars as the constituents of the cluster, and only brown dwarfs, very low mass stars, or white dwarfs are possible. If the constituents of the cluster are brown dwarfs, the collision timescale for our Plummer model in UGC 3789 is $t_{\text {coll }}<3.9 \times 10^{6}$ years, much less than the age of a galaxy. The timescale is even shorter if the constituent stars are mainsequence stars. However, if the cluster is composed of white dwarfs, the collision timescale can be as long as $1.8 \times 10^{7}$ years, but this is still much shorter than the lifetime of a galaxy. Therefore, we conclude that a compact cluster is not likely to survive long, and the dominant mass at the center of UGC 3789 is a supermassive BH. For all other megamaser galaxies except NGC 1194 and NGC 6264, the lifetimes of the central clusters are also shorter than the age of a galaxy (Table 4). The constraints on lifetime weakly rule out massive clusters in 
Table 6

Comparison of Maser BH Mass with Mass from Virial Estimation

\begin{tabular}{|c|c|c|c|c|c|c|c|}
\hline Galaxy & $\begin{array}{c}\text { Maser BH } \\
\left(10^{6} M_{\odot}\right)\end{array}$ & $\begin{array}{l}\text { Virial BH } \\
\left(10^{6} M_{\odot}\right) \\
\end{array}$ & $\begin{array}{c}R_{\mathrm{BLR}} \\
\text { (light-days) }\end{array}$ & $\begin{array}{c}V_{\mathrm{FWHM}} \\
\left(\mathrm{km} \mathrm{s}^{-1}\right)\end{array}$ & Reference & $\begin{array}{l}L_{X}(2-10 \mathrm{kev}) \\
\left(10^{42} \mathrm{erg} \mathrm{s}^{-1}\right) \\
\end{array}$ & Reference \\
\hline NGC 1068 & $8.6 \pm 0.3$ & $9.0 \pm 6.6$ & $4.6 \pm 3.0$ & 2900 & 1 & $6.5,2.6$ & 5,6 \\
\hline NGC 4388 & $8.5 \pm 0.2$ & $7.1 \pm 4.9$ & $2.0 \pm 1.2$ & 3900 & 2 & $0.8,0.9,1.9,1.0$ & $7,8,9,10$ \\
\hline NGC 2273 & $7.6 \pm 0.1$ & $4.3 \pm 2.8$ & $2.2 \pm 1.2$ & 2900 & 3 & $1.0,1.7$ & 11,12 \\
\hline Circinus & $1.7 \pm 0.3$ & $4.8 \pm 3.2$ & $1.9 \pm 1.1$ & 3300 & 4 & $1.1,1.0,1.2$ & $13,14,15$ \\
\hline
\end{tabular}

Notes. Column 1: galaxy name. Column 2: BH mass measured from the megamaser technique. The BH mass of NGC 1068 is taken from Lodato \& Bertin (2003) and Circinus from Greenhill et al. (2003). The BH masses of NGC 4388 and NGC 2273 are from this paper. Column 3: BH mass measured from the virial estimation method. Column 4: the size of the BLR calculated using the $L_{(2-10 \mathrm{kev})}-R_{\mathrm{BLR}}$ correlation (Kaspi et al. 2005). Column 5: the full width at half maximum (FWHM) of the observed broad line ( $\mathrm{H}_{\beta}$ for NGC 1068 and $\mathrm{H}_{\alpha}$ for NGC 4388, NGC 2273, and Circinus). In NGC 2273, only the full width at zero intensity (FWZI) is given, so we estimate the FWHM "by eye." We assume the measurement uncertainty is $200 \mathrm{~km} \mathrm{~s}^{-1}$ in all cases. Column 6: reference for the line width measurement. Column 7: intrinsic hard X-ray (2-10 kev) luminosity. We used the average luminosity to calculate the size of BLR. The error of the luminosity is taken to be the standard deviation of the adopted luminosities. Note that the distances used to calculate the intrinsic luminosities are 15.4, 19.0, 26.0, and 4.0 Mpc for NGC 1068, NGC 2273, NGC 4388, and Circinus, respectively. Column 8: reference for the adopted X-ray luminosities.

References. (1) Miller et al. (1991); (2) Ho et al. (1997); (3) Moran et al. (2000); (4) Oliva (1998); (5) Levenson et al. (2006); 6. Ogle et al. (2003); (7) Cappi et al. (2006); (8) Akylas \& Georgantopoulos (2009) 9. Bassani et al. (1999); (10) Forster et al. (1999); (11) Terashima et al. (2002); (12) Awaki et al. (2009); (13) Yang et al. (2009); (14) Matt et al. (1999); (15) Smith \& Wilson (2001).

NGC $4388\left(T_{\text {age }} \approx 7 \times 10^{9} \mathrm{yr}\right)$ but strongly rule out clusters in the others $\left(T_{\text {age }}<1.1 \times 10^{9} \mathrm{yr}\right)$.

In summary, by setting tight constraints on the sizes and central mass densities of possible Plummer clusters, we have been able to strongly rule out clusters as the dominant central masses in UGC 3079, NGC 2273, NGC 6323, and NGC 2960 and weakly rule out the clusters in NGC 4388. We argue that supermassive $\mathrm{BHs}$ are the dominant masses in these megamasers. Together with the Milky Way Galaxy, NGC 4258, and M31 (Kormendy 2004), the number of galaxies with strong evidence to rule out a massive star cluster as the dominant central mass increases from three to seven.

\section{COMPARISON WITH VIRIAL BH MASS ESTIMATES}

It is important to compare results from different methods for measuring $\mathrm{BH}$ masses, because such comparisons can provide insight into potential systematic errors for each method (Siopis et al. 2009; Kormendy 2004; Humphrey et al. 2009; Greene et al. 2010). Comparing optically measured dynamical $\mathrm{BH}$ masses with those from the $\mathrm{H}_{2} \mathrm{O}$ megamaser method is especially valuable, since the megamaser galaxies with Keplerian rotation curves provide the most direct and accurate $\mathrm{BH}$ mass measurements for external galaxies, These maser BH masses can be used to test the more commonly used $\mathrm{BH}$ mass measuring techniques in the optical, such as the stellar or gas dynamical methods.

One cannot meaningfully compare the stellar or gas dynamical BH masses with our maser BH masses unless the gravitational spheres of influence can be resolved. Otherwise, even if the optically determined dynamical masses agree with the maser $\mathrm{BH}$ masses within the errors, the uncertainty can be too large to tell the real accuracy of the stellar- or gas-dynamical method. The angular diameters of the spheres of influence of our maser BH masses, $2 R_{\text {inf }}$ (Table 4), range from 0.'006 to 0'.06. Among these megamasers, only the spheres of influence in NGC 4388 and NGC 1194 can be barely resolved by the HST (resolution $\approx 0{ }^{\prime} 07$ at $\lambda \approx 6500 \AA$ ) or the Very Large Telescope (VLT) assisted with adaptive optics (resolution $\approx 0^{\prime \prime} 1$ at $\lambda=2 \mu \mathrm{m}$ ). Therefore, of the galaxies here, stellar or gas dynamical measurements are feasible only for these two galaxies. We have obtained VLT time to measure the BH mass in NGC 4388, and we will apply the stellar dynamical method to this galaxy and compare the $\mathrm{BH}$ mass to the maser $\mathrm{BH}$ mass in the future.

Another commonly used BH mass measuring technique is the virial estimation method (Greene \& Ho 2006; Kim et al. 2008; Vestergaard \& Osmer 2009). We are able to compare this technique with the megamaser disk method in four galaxies, and this is the first time that the virial estimation method is directly tested. In this method, one estimates the BH mass as $M_{\mathrm{BH}}=f R_{\mathrm{BLR}} \sigma_{\text {line }}^{2} / G$, where $f$ is an unknown factor that depends on the structure, kinematics, and orientation of the broad-line region (BLR), $R_{\mathrm{BLR}}$ is the radius of the BLR, $\sigma_{\text {line }}$ is the gas velocity dispersion observed in the BLR, and $G$ is the gravitational constant. Here, we adopt the latest empirically determined $\langle f\rangle=5.2 \pm 1.3$ from Woo et al. (2010). Vestergaard (2009) suggests that the virial method is accurate to a factor of $\approx 4$. Since we cannot directly detect the BLRs in megamaser galaxies that have Seyfert 2 nuclei, we estimated $\sigma_{\text {line }}$ using the scattered "polarized broad lines" (PBL) from the hidden BLRs in those four megamaser galaxies with detected PBLs: NGC 1068, NGC 4388, NGC 2273, and Circinus. We estimated $R_{\mathrm{BLR}}$ via the $L_{(2-10 \mathrm{kev})}-R_{\mathrm{BLR}}$ correlation (Kaspi et al. 2005). Table 6 shows the resulting virial $\mathrm{BH}$ masses for these four galaxies.

Under the assumption that the observed line width of broad $\mathrm{H}_{\alpha}$ or $\mathrm{H}_{\beta}$ emission approximates the "intrinsic line width", ${ }^{14}$ we find that for NGC 1068, NGC 2273, NGC 4388, and Circinus, the $\mathrm{BH}$ masses at the $1 \sigma$ level measured by the virial estimation method agree within a factor of five with the megamaser $\mathrm{BH}$ masses. So, while our comparison is so far limited to these four galaxies, the BH mass measured from the virial method matches the megamaser BH mass to about the expected accuracy.

The application of the virial method to megamaser galaxies is described in detail in the Appendix.

\section{MASER BH MASSES AND THE $M-\sigma_{\star}$ RELATION}

Table 4 shows the $\mathrm{BH}$ masses for all seven megamaser galaxies. Along with the previously published $\mathrm{BH}$ masses from megamaser observations, it is interesting to see that the maser BH masses, except in Circinus, are within a factor of three of $2.2 \times 10^{7} M_{\odot}$, with the uncertainty of each $\mathrm{BH}$ mass

\footnotetext{
${ }^{14}$ By "intrinsic line width," we mean the line width one would measure if the
} BLRs were observed directly as in Type 1 AGNs. 
$\leqslant 12 \%$. This small range of masses could be a selection bias. Disk megamasers are preferentially detected in Seyfert 2 spiral galaxies, and since the local active $\mathrm{BH}$ mass function for Seyfert 2 galaxies peaks at $M_{\mathrm{BH}} \approx 3 \times 10^{7} M_{\odot}$ (Heckman et al. 2004), we may be limited to $\mathrm{BHs}$ in this range of masses by analyzing disk megamasers.

Our new maser BH masses more than double the number of galaxies having dynamical $\mathrm{BH}$ masses $M_{\mathrm{BH}} \sim 10^{7} M_{\odot}$. These measurements play a particularly important role in constraining the $M-\sigma_{\star}$ relation at the low-mass end of known nuclear $\mathrm{BH}$ masses. In a companion paper, Greene et al. (2010) find that the maser galaxies as a group fall significantly below the $M-\sigma_{\star}$ relation defined by more massive elliptical galaxies. As a result, the $M-\sigma_{\star}$ relation that fits later-type and lower-mass galaxies has both a larger scatter and lower zero point than the relation for elliptical galaxies alone. However, there is a potential caveat that measuring robust (well-defined) $\sigma_{\star}$ in late-type galaxies is challenging (Greene et al. 2010), and the contribution of systematic biases in $\sigma_{\star}$ to the deviation from the $M-\sigma_{\star}$ relation still needs to be explored in the future. With this caveat in mind, the observed deviations from the $M-\sigma_{\star}$ relation at low mass imply that the relation may not be a single, low-scatter power law as originally proposed, and our BH mass distribution is consistent with the best simulations of galaxy growth regulated by "radio" feedback (see Figure 4 of Croton et al. 2006).

In addition to our seven galaxies with maser BH masses, we currently have VLBI data sets for another four disk megamaser candidates, and we are monitoring the spectra of more than six disk megamaser candidates that are currently too faint to be observed with VLBI, but may flare up in the future. Along with these galaxies and more megamasers we may discover in the future with the GBT, we expect to obtain a larger sample of maser $\mathrm{BH}$ masses which could help to clarify the $M_{\mathrm{BH}}-\sigma_{\star}$ relation.

\section{SUMMARY}

Our main conclusions are the following.

1. The maser distributions in all seven megamaser galaxies are consistent with edge-on circumnuclear disks surrounding central massive objects in the AGNs. The inner radii of the disks are between 0.09 and $0.5 \mathrm{pc}$, similar to all previously published megamaser disks. The rotation curves of all seven megamaser disks are consistent with Keplerian rotation. Four of the megamaser disks reveal evidence for warps.

2. VLBI observations of circumnuclear megamaser disks are the only means to measure directly the enclosed mass and the mass density well within the radius of the gravitational sphere of influence of the central mass in these galaxies. The high central mass densities $\left((0.12-60) \times 10^{10} M_{\odot} \mathrm{pc}^{-3}\right)$ (within the central $0.3 \mathrm{pc}$ ) of the seven megamaser disks indicate that in all, except one, disks, the central mass is dominated by a supermassive $\mathrm{BH}$ rather than an extremely dense cluster of stars or stellar remnants.

3. The BH masses measured are all within a factor of three of $2.2 \times 10^{7} M_{\odot}$ and the accuracy of each BH mass is primarily limited by the accuracy of the Hubble constant. The narrow range of $\mathrm{BH}$ mass distribution may reflect selection from the local active-galaxy mass function.

4. Stellar dynamics cannot be applied to the majority of the megamasers presented here to measure $\mathrm{BH}$ masses with high precision. The gravitational spheres of influence in all cases except NGC 1194 and NGC 4388 are too small to be resolved by current optical telescopes. Observations with the VLT for measuring the BH mass in NGC 4388 with stellar-dynamical modeling are in progress.

5. Under the assumption that the broad emission line widths can be estimated from polarized scattered light, we have calibrated for the first time the $\mathrm{BH}$ mass determination by the virial estimation method based on optical observations. With the latest empirically determined $\langle f\rangle=5.2 \pm 1.3$, the virial estimated $\mathrm{BH}$ mass is within a factor of five at the $1 \sigma$ level of the accurate $\mathrm{BH}$ mass based on megamaser disks in NGC 1068, NGC 2273, NGC 4388, and Circinus. This is comparable to the factor of four accuracy expected for the virial estimation method.

6. The accurate $\mathrm{BH}$ masses in the seven megamaser galaxies contribute to the observational basis of the $M-\sigma_{\star}$ relation at the low-mass end. The deviation from the mean relation of the several accurate maser $\mathrm{BH}$ masses suggests that the relation may not be a single, low-scatter power law as originally proposed, which has interesting implications for the universality of the $M-\sigma_{\star}$ relation Greene et al. (2010).

The National Radio Astronomy Observatory is a facility of the National Science Foundation operated under cooperative agreement by Associated Universities, Inc. We thank Ed Fomalont for his kind help with our VLBI data reduction and Lincoln Greenhill for his substantial assistance for this project. C.Y.K. thanks Mark Whittle for his numerous insightful comments on our project. This research has made use of NASA's Astrophysics Data System Bibliographic Services, and the NASA/IPAC Extragalactic Database (NED) which is operated by the Jet Propulsion Laboratory, California Institute of Technology, under contract with the National Aeronautics and Space Administration.

\section{APPENDIX}

\section{APPLYING THE VIRIAL ESTIMATION METHOD TO MEGAMASER GALAXIES}

The virial estimation method for measuring $\mathrm{BH}$ masses in AGNs (e.g., Greene \& Ho (2006), Kim et al. (2008), Vestergaard \& Osmer (2009)) uses the BLR gas as a dynamical tracer. It is usually only applied to Type 1 AGNs, where the BLRs can be directly observed. In this method, one estimates the $\mathrm{BH}$ mass with the following equation:

$$
M_{\bullet}=\frac{f R_{\mathrm{BLR}} \sigma_{\text {line }}^{2}}{\mathrm{G}},
$$

where $f, R_{\mathrm{BLR}}$, and $\sigma_{\text {line }}$ have been defined in Section 5. Since one can directly detect light from the BLRs in Type 1 AGNs, $\sigma_{\text {line }}$ can be measured from the broad line spectra and one can use the continuum luminosity $\lambda L_{\lambda}(5100 \AA)$ to estimate $R_{\mathrm{BLR}}$ via the $\lambda L_{\lambda}(5100 \AA)-R_{\mathrm{BLR}}$ correlation (e.g., Kaspi et al. 2000, 2005).

In a Type $2 \mathrm{AGN}$, including the megamaser galaxies we study here, our line-of-sight to the BLR is blocked by heavy dust extinction, so one cannot directly measure $\lambda L_{\lambda}(5100 \AA)$ and $\sigma_{\text {line }}$. Instead, one can probe the BLRs in megamaser galaxies with polarized scattered light and hard X-rays. Among all the megamaser galaxies with measured $\mathrm{BH}$ masses, polarized scattered light from the BLRs has been detected in NGC 1068, NGC 4388, NGC 2273, and Circinus (see Table 6), and X-ray measurements are also available for these four galaxies. 
We can estimate $\sigma_{\text {line }}$ from the relation $\sigma_{\text {line }} / V_{\mathrm{FWHM}}=$ $2.09 \pm 0.45$ from Woo et al. (2010), where $V_{\text {FWHM }}$ is the FWHM width of polarized scattered light in the broad $\mathrm{H}_{\alpha}$ line (NGC 2273, NGC 4388, and Circinus) or $\mathrm{H}_{\beta}$ line (NGC 1068). The major concern with these line widths is that the observed values may not be the same as the line width one would measure if the BLRs could be observed directly. We identify two effects that can induce such a difference from the well-studied case NGC 1068 (Miller et al. 1991). First, the broad $\mathrm{H}_{\beta}$ lines in the polarized flux spectra can contain a contribution from the narrow $\mathrm{H}_{\beta}$ lines. Without removing the contribution from narrow lines, the BLR line width may be underestimated by $\sim 20 \%-30 \%$ in NGC 1068. Second, the polarized emission from AGNs may originate from light being scattered by electrons with a temperature a few times $10^{5} \mathrm{~K}$, which results in significant thermal broadening $(\sim 50 \%)$ of the spectral lines. Because of these two effects, there will be systematic errors in $\sigma_{\text {line }}$ if one directly uses the observed line width of the polarized lines to estimate $\sigma_{\text {line }}$. Among the four megamaser galaxies we consider here, only NGC 1068 has been studied in enough detail to remove these two effects. Luckily, the two effects change the line width in opposite directions, and hence could offset each other to a certain extent. As in NGC 1068, if no correction is made for these two effects, the systematic error will be only $\sim 10 \%$, just slightly larger than the measurement error. Without knowing the actual contributions of these two effects for the other three galaxies, we assume that the two effects cancel each other to the same extent as in NGC 1068 and use the observed line widths as the approximations for the intrinsic widths. The reader should be aware of this caveat when interpreting the comparisons made.

In this work, $R_{\mathrm{BLR}}$ was estimated from the "intrinsic" $L_{(2-10 \mathrm{kev})}$ of the nuclear region via the $L_{(2-10 \mathrm{kev})}-R_{\mathrm{BLR}}$ correlation (Kaspi et al. 2005). Since three (NGC 1068, NGC 2273, and Circinus) of the four megamaser galaxies considered here are Compton-thick (i.e., the X-ray absorbing column density is $\left.>10^{24} \mathrm{~cm}^{-2}\right)$, we paid particular attention to how the $L_{(2-10 \mathrm{kev})}$ were measured. The Compton thick nature of these AGNs is a problem because the intrinsic radiation is mostly suppressed and the X-ray spectrum is dominated by the reflected or scattered components. It is difficult to measure the actual absorbing column density and it is very likely that the intrinsic hard $\mathrm{X}$-ray luminosity is severely underestimated (e.g., Levenson et al. 2006; Bassani et al. 1999). Therefore, we excluded those measurements that did not consider the Compton-thick nature of these sources and failed to give the absorbing column density in the expected range. We mainly considered those measurements from either data with appropriate modeling or from observations with instruments capable of directly measuring the transmission components of X-ray above $10 \mathrm{keV}$. We took at least two different measurements for each galaxy from the literature and used the average value to calculate $R_{\mathrm{BLR}}$ from the correlation in Kaspi et al. (2005).

Given $R_{\mathrm{BLR}}$ and $\sigma_{\text {line }}$, we estimated the $\mathrm{BH}$ masses using Equation (A1) with the empirically determined $\langle f\rangle=5.2_{-1.3}^{+1.3}$ from Woo et al. (2010). The resultant BH masses were compared with the maser BH masses in Section 5.

\section{REFERENCES}

Akylas, A., \& Georgantopoulos, I. 2009, A\&A, 500, 999

Argon, A. L., Greenhill, L. J., Reid, M. J., Moran, J. M., \& Humphreys, E. M. L. 2007, ApJ, 659, 1040

Awaki, H., Terashima, Y., Higaki, Y., \& Fukazawa, Y. 2009, PASJ, 61, 317
Barth, A. J. 2004, in Carnegie Observatories Astrophysics Series, Vol. 1 Coevolution of Black Holes and Galaxies, ed. L. C. Ho (Pasadena, CA: Carnegie Observatories), 21

Bassani, L., Dadina, M., Maiolino, R., Salvati, M., Risaliti, G., Della Ceca, R., Matt, G., \& Zamorani, G. 1999, ApJS, 121, 473

Binney, J., \& Tremaine, S. 2008, Galactic Dynamics (Princeton, NJ: Princeton Univ. Press)

Braatz, J., Condon, J., Reid, M., Henkel, C., Lo, K. Y., Kuo, C. Y., Impellizzeri, C., \& Hao, L. 2009, The Megamaser Cosmology Project Large Proposal, submitted to NRAO June, 1, 2009

Braatz, J. A., Reid, M. J., Humphreys, E. M. L., Henkel, C., Condon, J. J., \& Lo, K. Y. 2010, ApJ, 718, 657

Cappi, M., et al. 2006, A\&A, 446, 459

Croton, D. J., et al. 2006, MNRAS, 365, 11

Ferrarese, L., \& Ford, H. 2005, SSRv, 116, 523

Ferrarese, L., \& Merritt, D. 2000, ApJ, 539, 9

Forster, K., Leighly, K. M., \& Kay, L. E. 1999, ApJ, 523, 521

Freedman, W. L., \& Madore, B. F. 2010, ARA\&A, 48, 673

Gebhardt, K., et al. 2000, ApJ, 539, 13

Green, J. E., \& Ho, L. C. 2006, ApJ, 641, 21

Greene, J. E., et al. 2010, ApJ, 721, 26

Greenhill, L. J., Gwinn, C. R., Antonucci, R., \& Barvainis, R. 1996, ApJ, 472, 21

Greenhill, L. J., Moran, J. M., \& Herrnstein, J. R. 1997, ApJ, 481, 23

Greenhill, L. J., Moran, J. M., Reid, M. J., Menten, K. M., \& Hirabayashi, H. 1993, ApJ, 406, 482

Greenhill, L. J., et al. 2003, ApJ, 590, 162

Gültekin, K., et al. 2009, ApJ, 698, 198

Heckman, T. M., Kauffmann, G., Brinchmann, J., Charlot, S., Tremonti, C., \& White, S. D. M. 2004, ApJ, 613, 109

Henkel, C., Braatz, J. A., Greenhill, L. J., \& Wilson, A. S. 2002, A\&A, 394, 23

Herrnstein, J. R., et al. 1999, Nature, 400, 539

Ho, L. C., Filippenko, A. V., Sargent, W. L. W., \& Peng, C. Y. 1997, ApJS, 112, 391

Humphrey, P. J., Buote, D. A., Brighenti, F., Gebhardt, K., \& Mathews, W. G. 2009, ApJ, 703, 1257

Humphreys, E. M. L., Reid, M. J., Greenhill, L. J., Moran, J. M., \& Argon, A. L. 2008, ApJ, 672, 800

Kaspi, S., Maoz, D., Netzer, H., Peterson, B. M., Vestergaard, M., \& Jannuzi, B. T. 2005, ApJ, 629, 61

Kaspi, S., Smith, P. S., Netzer, H., Maoz, D., Jannuzi, B. T., \& Giveon, U. 2000, ApJ, 533, 631

Kim, M., Ho, L. C., Peng, C. Y., Barth, A. J., Im, M., Martini, P., \& Nelson, C. H. 2008, ApJ, 687, 767

Kondratko, P. T., Greenhill, L. J., \& Moran, J. M. 2005, ApJ, 618, 618

Kondratko, P. T., Greenhill, L. J., \& Moran, J. M. 2008, ApJ, 678, 87

Kormendy, J. 2004, in Carnegie Observatories Astrophysics Series, Vol. 1, Coevolution of Black Holes and Galaxies, ed. L. C. Ho (Cambridge: Cambridge Univ. Press), 1

Kormendy, J., \& Gebhardt, K. 2001, in AIP Conf. Proc. 586, Relativistic Astrophysics: 20th Texas Symposium, ed. J. C Wheeler \& H. Martel (Melville, NY: AIP), 363

Kormendy, J., \& Richstone, D. 1995, ARA\&A, 33, 581

Kukula, M. J., Pedlar, A., Baum, S. A., \& O'Dea, C. P. 1995, MNRAS, 276, 1262

Levenson, N. A., Heckman, T. M., Krolik, J. H., Weaver, K. A., \& Życki, P. T. 2006, ApJ, 648, 111

Lo, K. Y. 2005, ARA\&A, 43, 625

Lodato, G., \& Bertin, G. 2003, A\&A, 398, 517

Lu, N. Y., Hoffman, G. L., Groff, T., Roos, T., \& Lamphier, C. 1993, ApJS, 88, 383

Mamyoda, K., Nakai, N., Yamauchi, A., Diamond, P., \& Huré, J.-M. 2009, PASJ, 61, 1143

Maoz, E. 1995, ApJ, 455, 131

Maoz, E. 1998, ApJ, 494, 181

Matt, G., et al. 1999, A\&A, 341, 39

Miller, J. S., Goodrich, R. W., \& Mathews, W. G. 1991, ApJ, 378, 47

Mioduszewski, A., \& Kogan, L. 2000, AIPS Memo 110, http://www.aips.nrao. edu/aipsmemo.html

Moran, E. C., Barth, A. J., Kay, L. E., \& Filippenko, A. V. 2000, ApJ, 540, 73

Ogle, P. M., Brookings, T., Canizares, C. R., Lee, J. C., \& Marshall, H. L. 2003, A\&A, 402, 849

Oliva1, E., Marconi, A., Cimatti, A., \& di Serego Alighieri, S. 1998, A\&A, 329, 21

Plummer, H. C. 1915, MNRAS, 76, 107

Reid, M. J., Braatz, J. A., Condon, J. J., Greenhill, L. J., Henkel, C., \& Lo, K. Y. 2009a, ApJ, 695, 287 
Reid, M. J., Menten, K. M., Brunthaler, A., Zheng, X. W., Moscadelli, L., \& Xu, Y. 2009b, ApJ, 693, 397

Richstone, D., et al. 1998, Nature, 395, A14

Riess, A. G., et al. 2009, ApJ, 699, 539

Russell, D. G. 2002, ApJ, 565, 681

Siopis, C., et al. 2009, ApJ, 693, 946

Smith, D. A., \& Wilson, A. S. 2001, ApJ, 557, 180

Terashima, Y., Iyomoto, N., Ho, L. C., \& Ptak, A. F. 2002, ApJS, 139, 1
Vestergaard, M. 2009, in STScI Spring Symposium 2007 on Black Holes, ed. A. Koekemoer (Cambridge: Cambridge Univ. Press), in press (arXiv:0904.2615)

Vestergaard, M., \& Osmer, P. S. 2009, ApJ, 699, 800

Walker, C., \& Chatterjee, S. 2000, VLBA Scientific Memo 23, http://www.vlba.nrao.edu/memos/sci/

Woo, J.-H., et al. 2010, ApJ, 716, 269

Yang, Y., Wilson, A. S., Matt, G., Terashima, Y., \& Greenhill, L. J. 2009, ApJ, 691,131 